\title{
Fundamental groups of moduli stacks of stable curves of compact type
}

\author{
MARCO BOGGI
}

Let $\widetilde{\mathcal{M}}_{g, n}$, for $2 g-2+n>0$, be the moduli stack of $n$-pointed, genus $g$, stable complex curves of compact type. Various characterizations and properties are obtained of both the topological and algebraic fundamental groups of the stack $\widetilde{\mathcal{M}}_{g, n}$. For instance, in Theorem 3.20, we show that the topological fundamental groups are linear, extending to all $n \geq 0$ previous results of Morita and Hain for $g \geq 2$ and $n=0,1$.

Let $\Gamma_{g, n}$, for $2 g-2+n>0$, be the Teichmüller group associated with a compact Riemann surface of genus $g$ with $n$ points removed $S_{g, n}$, ie the group of homotopy classes of diffeomorphisms of $S_{g, n}$ which preserve the orientation of $S_{g, n}$ and a given order of its punctures. Let $\mathcal{K}_{g, n}$ be the normal subgroup of $\Gamma_{g, n}$ generated by Dehn twists along separating simple closed curves (briefly s.c.c.) on $S_{g, n}$. The above theory yields a characterization of $\mathcal{K}_{g, n}$ for all $n \geq 0$, improving Johnson's classical results for closed and one-punctured surfaces in [13].

The Torelli group $\mathcal{T}_{g, n}$ is the kernel of the natural representation $\Gamma_{g, n} \rightarrow \operatorname{Sp}_{2 g}(\mathbb{Z})$. The abelianization of the Torelli group $\mathcal{T}_{g, n}$ is determined for all $g \geq 1$ and $n \geq 1$, thus completing classical results of Johnson [14] and Mess [18] for closed and one-punctured surfaces.

We also prove that a connected finite étale cover $\widetilde{\mathcal{M}}^{\lambda}$ of $\widetilde{\mathcal{M}}_{g, n}$, for $g \geq 2$, has a Deligne-Mumford compactification $\overline{\mathcal{M}}^{\lambda}$ with finite fundamental group. This implies that, for $g \geq 3$, any finite index subgroup of $\Gamma_{g}$ containing $\mathcal{K}_{g}$ has vanishing first cohomology group, improving a result of Hain [8].

$32 \mathrm{G} 15 ; 14 \mathrm{H} 10,30 \mathrm{~F} 60,14 \mathrm{~F} 35$

\section{Introduction}

Let $\mathcal{M}_{g, n}$ be the moduli stack of smooth, $n$-pointed, genus $g$, complex curves and let $\overline{\mathcal{M}}_{g, n}$ be its Deligne-Mumford compactification, ie the moduli stack of stable, $n$-pointed, genus $g$, complex curves. They are actually Deligne-Mumford stacks (briefly $D-M$ stacks). 
A central role in the study of the space $\mathcal{M}_{g, n}$ is played by the Teichmüller group $\Gamma_{g, n}$. Classically, it is defined, for a given compact Riemann surface of genus $g$ with $n$ points removed $S_{g, n}$, as the mapping class group of $S_{g, n}$, ie the group of homotopy classes of diffeomorphisms of $S_{g, n}$ which preserve the orientation of $S_{g, n}$ and a given order of its punctures. From our point of view, the most significant characterization of $\Gamma_{g, n}$ is as the fundamental group of the topological stack underlying $\mathcal{M}_{g, n}$.

Since, topologically, the space $\mathcal{M}_{g, n}$ is an Eilenberg-MacLane space of type $(\pi, 1)$, in principle, all topological properties of $\mathcal{M}_{g, n}$ can be derived from the study of $\Gamma_{g, n}$. As shown in Boggi [5], also many interesting algebro-geometric properties are encoded in the algebraic fundamental group of $\mathcal{M}_{g, n}$, ie in the profinite completion of $\Gamma_{g, n}$. According to the anabelian philosophy of Grothendieck, much more is to be expected when one takes in Galois actions. All this undoubtedly accounts for the extraordinary richness and difficulty of Teichmüller theory.

At the other extreme lies the case of the D-M stack $\overline{\mathcal{M}}_{g, n}$, whose fundamental group is trivial (see Proposition 1.1 in Boggi and Pikaart [6]).

The purpose of this paper is a systematic study of the topological and algebraic fundamental group of a partial compactification of $\mathcal{M}_{g, n}$, contained as an open substack in the Deligne-Mumford compactification $\overline{\mathcal{M}}_{g, n}$, which is, in some sense, intermediate between the two. Let $\widetilde{\mathcal{M}}_{g, n}$ be the moduli stack of $n$-pointed, genus $g$ stable complex curves of compact type. In other words, the stack $\widetilde{\mathcal{M}}_{g, n}$ parametrizes stable curves which have a compact generalized jacobian or, equivalently, whose dual graph is a tree.

The topological and algebraic fundamental groups of $\widetilde{\mathcal{M}}_{g, n}$ are characterized in various ways. Let $\mathcal{K}_{g, n}$ be the normal subgroup of $\Gamma_{g, n}$ generated by Dehn twists along separating s.c.c. on $S_{g, n}$. The first and almost trivial characterization of the fundamental group of $\widetilde{\mathcal{M}}_{g, n}$ is as the quotient of the mapping class group $\Gamma_{g, n}$ by $\mathcal{K}_{g, n}$. Eventually, it is proved that the fundamental group of $\widetilde{\mathcal{M}}_{g, n}$ is a linear group and, more precisely, an extension of the symplectic group $\mathrm{Sp}_{2 g}(\mathbb{Z})$ by a free abelian group of rank $2(n-1)$, for $g=1$ and $n \geq 1$, and of rank $\left(\begin{array}{c}2 g \\ 3\end{array}\right)+2 g(n-1)$, for $g \geq 2$. This is a generalization of previous results by Morita [21] and Hain [9] who dealt with the cases $g \geq 2$ and $n=0,1$. In particular, it turns out to be a much simpler object than the Teichmüller group itself. It is important to stress that, in contrast with the usual situation, sometimes the characterizations of the topological fundamental group are derived from those of the algebraic one, as it happens for instance in the proof of Theorem 3.15.

The above theory turns out to be very useful because, even though the space $\widetilde{\mathcal{M}}_{g, n}$ in general is not an Eilenberg-MacLane space of type $(\pi, 1)$ (see Proposition A.4 in Mondello [20]) its fundamental group still contains a lot of information on the moduli stack $\widetilde{\mathcal{M}}_{g, n}$ and on the topology of its open substack $\mathcal{M}_{g, n}$ as well. This will be 
apparent from the applications given in Boggi [5] to the monodromy of families of curves of compact type. As to $\mathcal{M}_{g, n}$, let us mention Proposition 5.1 and Remark 5.2.

However, the most important applications, given in the present paper, of the above results are to some questions in classical Teichmüller theory. For $n=0$ and $g \geq 2$, Johnson characterized $\mathcal{K}_{g, n}$ as the kernel of the natural representation:

$$
\Gamma_{g} \rightarrow \operatorname{Out}\left(\pi_{1}\left(S_{g}\right) / \pi_{1}\left(S_{g}\right)^{[3]}\right),
$$

where $\pi_{1}\left(S_{g}\right)^{[3]}$ is the third term of the descending central series of the fundamental group of $S_{g}$. From the point of view of Hodge theory, the weight filtration $W^{k} \pi_{1}\left(S_{g, n}\right)$, for $k \geq 0$, (see Section 2 for the definition) is the natural extension of the descending central series to the $n$-punctured case. In Theorem 3.15, it is proved that, for $2 g-2+n>$ 0 , the group $\mathcal{K}_{g, n}$ is the kernel of the natural representation:

$$
\Gamma_{g, n} \rightarrow \operatorname{Out}\left(\pi_{1}\left(S_{g, n}\right) / W^{3} \pi_{1}\left(S_{g, n}\right)\right) .
$$

Let $\mathcal{T}_{g, n}$ be the Torelli subgroup of $\Gamma_{g, n}$. It is defined as the kernel of the natural representation $\Gamma_{g, n} \rightarrow \mathrm{Sp}_{2 g}(\mathbb{Z})$, ie as the subgroup of mapping classes of $\Gamma_{g, n}$ acting trivially on the homology of the compact surface $S_{g}$. In the series of papers [11; 12 ; 13; 14], Johnson computed the abelianization of $\mathcal{T}_{g, n}$ for $g \geq 3$ and $n=0,1$, while Mess [18] computed the abelianization of $\mathcal{T}_{2}$. Then in [9], Hain and Looijenga implemented their results in order to compute $H_{1}\left(\mathcal{T}_{g, n}, \mathbb{Q}\right)$ for all $g \geq 1$ and $n \geq 1$. Thanks to the above characterization of the group $\mathcal{K}_{g, n}$, the above results are completed, determining the abelianization of the Torelli group $\mathcal{T}_{g, n}$ for all $g \geq 1$ and $n \geq 1$ (Theorem 3.21).

Finally, let us mention a result which, in the light of Boggi [5], is also interesting. For $g_{1}$ and $g_{2}$ nonnegative integers, such that $g_{1}+g_{2}=g$, and a partition of the set of the $n$ marking labels in two subsets of cardinality $n_{1}$ and $n_{2}$, Knudsen [16] defined a clutching morphism $\overline{\mathcal{M}}_{g_{1}, n_{1}+1} \times \overline{\mathcal{M}}_{g_{2}, n_{2}+1} \rightarrow \overline{\mathcal{M}}_{g, n}$ which, on the moduli space of stable curves of compact type, restricts to a morphism $\widetilde{\mathcal{M}}_{g_{1}, n_{1}+1} \times \widetilde{\mathcal{M}}_{g_{2}, n_{2}+1} \rightarrow \widetilde{\mathcal{M}}_{g, n}$. In Theorem 4.1, it is proved that such morphism induces a monomorphism between the respective topological fundamental groups (for the algebraic fundamental groups the situation is a little bit trickier, due to the failure of the congruence subgroup property for $\mathrm{SL}_{2}(\mathbb{Z})$ ).

\section{Level structures over moduli of curves}

The purpose of this section is, basically, to provide the notation to be used in the rest of the paper. For a more complete treatment of level structures and Teichmüller theory, we refer the reader, for instance, to Section 1 of Boggi [4]. 
Let $\overline{\mathcal{M}}_{g, n}$, for $2 g-2+n>0$, be the stack of $n$-pointed, genus $g$, stable algebraic curves over $\mathbb{C}$. It is a regular connected proper D-M stack over $\mathbb{C}$ of dimension $3 g-3+n$, and it contains, as an open substack, the stack $\mathcal{M}_{g, n}$ of $n$-pointed, genus $g$, smooth algebraic curves over $\mathbb{C}$. By Proposition 1.1 in Boggi and Pikaart [6], the stack $\overline{\mathcal{M}}_{g, n}$ is simply connected. On the contrary, the stack $\mathcal{M}_{g, n}$ has plenty of nontrivial covers which we are briefly going to introduce in this section. Its universal cover, the Teichmüller space $T_{g, n}$, is a contractible complex manifold. There is a natural way to define homotopy groups for topological D-M stacks, as done, for instance, by Noohi in $[24 ; 23]$. Then, the fundamental group $\pi_{1}\left(\mathcal{M}_{g, n}, a\right)$ can be identified with the Teichmüller modular group $\Gamma_{g, n}$, which is the deck transformation group of the cover $T_{g, n} \rightarrow \mathcal{M}_{g, n}$.

A level structure $\mathcal{M}^{\lambda}$ is a finite, connected, Galois, étale cover of the stack $\mathcal{M}_{g, n}$ (by étale cover, we mean here an étale, surjective, representable morphism of algebraic stacks), therefore it is also represented by a regular D-M stack $\mathcal{M}^{\lambda}$. The level associated to $\mathcal{M}^{\lambda}$ is the finite index normal subgroup $\Gamma^{\lambda}:=\pi_{1}\left(\mathcal{M}^{\lambda}, a^{\prime}\right)$ of the Teichmüller group $\Gamma_{g, n}$.

A level structure $\mathcal{M}^{\lambda^{\prime}}$ dominates $\mathcal{M}^{\lambda}$, if there is a natural étale morphism $\mathcal{M}^{\lambda^{\prime}} \rightarrow \mathcal{M}^{\lambda}$ or, equivalently, $\Gamma^{\lambda^{\prime}} \leq \Gamma^{\lambda}$. To mark the fact that $\mathcal{M}^{\lambda}$ is a level structure over $\mathcal{M}_{g, n}$, we will often denote it by $\mathcal{M}_{g, n}^{\lambda}$.

The morphism $p: \mathcal{M}_{g, n+1} \rightarrow \mathcal{M}_{g, n}$, forgetting the last label, is naturally isomorphic to the universal curve over $\mathcal{M}_{g, n}$. One can then identify the fiber $p^{-1}(a)$ with an $n$-punctured, genus $g$ curve $C \backslash\left\{P_{1}, \ldots, P_{n}\right\}$, where $\left(C, P_{1}, \ldots, P_{n}\right)$ is a curve in the class determined by $a \in \overline{\mathcal{M}}_{g, n}$. Denote by $S_{g, n}$ the Riemann surface underlying $C \backslash\left\{P_{1}, \ldots, P_{n}\right\}$ and fix a point $\tilde{a} \in S_{g, n}$. Since $p$ is a Serre fibration and $\pi_{2}\left(\mathcal{M}_{g, n}\right)=$ $\pi_{2}\left(T_{g, n}\right)=0$, there is a short exact sequence on fundamental groups

$$
1 \rightarrow \pi_{1}\left(S_{g, n}, \widetilde{a}\right) \rightarrow \pi_{1}\left(\mathcal{M}_{g, n+1}, \widetilde{a}\right) \rightarrow \pi_{1}\left(\mathcal{M}_{g, n}, a\right) \rightarrow 1 .
$$

By a standard argument this defines a monodromy representation:

$$
\rho: \pi_{1}\left(\mathcal{M}_{g, n}, a\right) \rightarrow \operatorname{Out}\left(\pi_{1}\left(S_{g, n}, \widetilde{a}\right)\right),
$$

called the universal monodromy representation. From the above fibration, it follows also that there is a natural representation of $\pi_{1}\left(\mathcal{M}_{g, n}, a\right)$ in the group of homotopy classes of self-homeomorphism of the Riemann surface $S_{g, n}$. Let us denote by $\mathrm{Hom}^{+}\left(S_{g, n}\right)$ the subgroup of orientation preserving self-homeomorphism of $S_{g, n}$ and by $\operatorname{Hom}^{0}\left(S_{g, n}\right)$ the subgroup consisting of homeomorphism homotopic to the identity. The Teichmüller modular group is classically defined to be the group of homotopy classes of homeomorphism of $S_{g, n}$ which preserve the orientation and the 
given order of the punctures:

$$
\Gamma_{g, n}:=\operatorname{Hom}^{+}\left(S_{g, n}\right) / \operatorname{Hom}^{0}\left(S_{g, n}\right),
$$

where $\operatorname{Hom}_{0}\left(S_{g, n}\right)$ is the connected component of the identity in the topological group of homeomorphisms $\operatorname{Hom}^{+}\left(S_{g, n}\right)$. By Teichmüller theory, the monodromy representation $\rho$ is faithful and identifies the fundamental group $\pi_{1}\left(\mathcal{M}_{g, n}, a\right)$ with the Teichmüller group $\Gamma_{g, n}$.

Let us denote by $\Pi_{g, n}$ the fundamental group of $S_{g, n}$ based in $\widetilde{a}$ and give $\Pi_{g, n}$ the standard presentation:

$$
\Pi_{g, n}=\left\langle\alpha_{1}, \ldots \alpha_{g}, \beta_{1}, \ldots, \beta_{g}, u_{1}, \ldots, u_{n} \mid \prod_{i=1}^{g}\left[\alpha_{i}, \beta_{i}\right] \cdot u_{n} \cdots u_{1}\right\rangle,
$$

where $u_{i}$, for $i=1, \ldots, n$, is a simple loop around the puncture $P_{i}$. For $n \geq 1$, let $A(g, n)$ be the group of automorphisms of $\Pi_{g, n}$ which fix the conjugacy classes of all $u_{i}$. For $n=0$, let instead $A(g, 0)$ be the image of $A(g, 1)$ in the automorphism group of $\Pi_{g}:=\Pi_{g, 0}$. Finally, let $I(g, n)$ be the group of inner automorphisms of $\Pi_{g, n}$. With this notation, the representation $\rho$ is faithful and gives a natural isomorphism $\Gamma_{g, n} \cong A(g, n) / I(g, n)$.

The most natural way to define levels is provided by the above isomorphism. In general, for a subgroup $\Pi^{\lambda} \leq \Pi_{g, n}$, which is invariant under $A(g, n)$ (in such case, we simply say that $\Pi^{\lambda}$ is invariant), it is defined a representation:

$$
\rho_{\lambda}: \Gamma_{g, n} \rightarrow \operatorname{Out}\left(\Pi_{g, n} / \Pi^{\lambda}\right)
$$

whose kernel we denote by $\Gamma^{\lambda}$. When $\Pi^{\lambda}$ has finite index in $\Pi_{g, n}$, then $\Gamma^{\lambda}$ has finite index in $\Gamma_{g, n}$ and is called the geometric level associated to $\Pi^{\lambda}$. The corresponding level structure is denoted by $\mathcal{M}_{g, n}^{\lambda}$.

A class of finite index invariant subgroups of the group $\Pi_{g, n}$ one can consider, in order to define geometric level structures, is that obtained from the descending central series, twisting by $l$-th powers. The descending central series is defined by $\Pi^{[1]}:=\Pi_{g, n}$ and $\Pi^{[k]}:=\left[\Pi^{[k-1]}, \Pi\right]$. Let then $\Pi^{l}$ be the invariant subgroup of $\Pi_{g, n}$ spanned by $l$-th powers and define

$$
\Pi^{[k], l}:=\Pi^{[k]} \cdot \Pi^{l} .
$$

We denote the corresponding levels and level structures by $\Gamma^{[k], l}$ and $\mathcal{M}^{[k], l}$.

Even though algebraically, the above filtration is very natural, from a geometric point of view, the so called weight filtration is more significative. This filtration originates in 
Hodge theory and is defined as follows. Let $N$ be the kernel of the natural morphism $\Pi_{g, n} \rightarrow \Pi_{g, 0}$, filling in the punctures, and define

$$
\begin{array}{ll}
W^{1} \Pi \quad:=\Pi_{g, n}, \\
W^{2} \Pi \quad:=N \cdot \Pi^{[2]}, \\
W^{k+1} \Pi:=\left[\Pi_{g, n}, W^{k} \Pi\right] \cdot\left[N, W^{k-1} \Pi\right] .
\end{array}
$$

As for the descending central series, one has $\left[W^{s} \Pi, W^{t} \Pi\right] \leq W^{s+t} \Pi$. The descending central series and the weight filtration are cofinal to each other and coincide for $n=0$. Indeed, $W^{2 k-1} \Pi \leq \Pi^{[k]} \leq W^{k} \Pi$. As above, let us define

$$
W^{k, l} \Pi=W^{k} \Pi \cdot \Pi^{l} .
$$

The corresponding representation is denoted by $\rho_{w(k, l)}$ and the corresponding levels and level structures are denoted by $\Gamma^{w(k, l)}$ and $\mathcal{M}^{w(k, l)}$ respectively. The kernel of the representation $\rho_{W^{k}}: \Gamma_{g, n} \rightarrow \operatorname{Out}\left(\Pi_{g, n} / W^{k} \Pi\right)$ will be instead denoted by $W^{k} \Gamma$.

Of particular interest are the levels defined by the kernels of the representations:

$$
\rho_{(m)}: \Gamma_{g, n} \rightarrow \operatorname{Sp}\left(H_{1}\left(S_{g}, \mathbb{Z} / m\right)\right), \quad \text { for } m \geq 2 .
$$

They are denoted by $\Gamma(m)$ and called abelian levels of order $m$. The corresponding level structures are then denoted by $\mathcal{M}^{(m)}$.

The kernel of the representation $\Gamma_{g, n} \rightarrow \mathrm{Sp}_{2 g}(\mathbb{Z})$ is denoted by $\mathcal{T}_{g, n}$ and called the Torelli subgroup of $\Gamma_{g, n}$. Note that $\Gamma(m)=\Gamma^{w(2, m)}$ and $\mathcal{T}_{g, n}=W^{2} \Gamma$.

The usual way to compactify a level structure $\mathcal{M}^{\lambda}$ over $\mathcal{M}_{g, n}$ is to take the normalization of $\overline{\mathcal{M}}_{g, n}$ in the function field of $\mathcal{M}^{\lambda}$. A more functorial definition can be given in the category of log regular schemes as done by Mochizuki in [19]. Let $\partial$ be the logarithmic structure on $\overline{\mathcal{M}}_{g, n}$ associated to the normal crossing divisor $\partial \mathcal{M}:=\overline{\mathcal{M}}_{g, n} \backslash \mathcal{M}_{g, n}$. We define a level structure over $\left(\overline{\mathcal{M}}_{g, n}, \partial\right)$ to be a finite, connected, log étale cover

$$
\left(\overline{\mathcal{M}}^{\lambda}, \partial^{\lambda}\right) \rightarrow\left(\overline{\mathcal{M}}_{g, n}, \partial\right)
$$

Then, by the $\log$ purity theorem, any level structure $\mathcal{M}^{\lambda}$ over $\mathcal{M}_{g, n}$ admits a canonical compactification to a level structure $\left(\overline{\mathcal{M}}^{\lambda}, \partial^{\lambda}\right)$ over $\left(\overline{\mathcal{M}}_{g, n}, \partial\right)$, where $\overline{\mathcal{M}}^{\lambda}$ is the normalization of $\overline{\mathcal{M}}_{g, n}$ in the function field of $\mathcal{M}^{\lambda}$ and $\partial^{\lambda}$ the logarithmic structure associated to the normal crossing divisor $\partial \mathcal{M}^{\lambda}:=\overline{\mathcal{M}}^{\lambda}-\mathcal{M}^{\lambda}$ (the Deligne-Mumford boundary of $\overline{\mathcal{M}}^{\lambda}$ ). On the other hand, it is also clear that any level structure over $\left(\overline{\mathcal{M}}_{g, n}, \partial\right)$ can be realized in this way. So, forgetting the logarithmic structure, one is back to the previous definition. A basic property of (compactified) level structures is the following (see, for instance, Deligne [7]): 
Proposition 2.1 If a level $\Gamma^{\lambda}$ is contained in an abelian level of order $m$, for some $m \geq 3$, then the level structure $\overline{\mathcal{M}}^{\lambda}$ is represented by a projective variety.

There is a very explicit and elementary method to describe the compactifications $\overline{\mathcal{M}}^{\lambda}$, locally in the analytic topology. A neighborhood of a point $a \in \mathcal{M}^{\lambda}$ is just the base of the local universal deformation of the fiber $C_{a}$ in $a$ of the universal family $\mathcal{C}^{\lambda} \rightarrow \mathcal{M}^{\lambda}$. Let us see how a neighborhood of $a \in \partial \mathcal{M}^{\lambda}$ can be described.

Let $\mathcal{B} \rightarrow \overline{\mathcal{M}}_{g, n}$ be an analytic neighborhood of the image $y$ of $a$ in $\overline{\mathcal{M}}_{g, n}$ such that:

- local coordinates $z_{1}, \ldots, z_{3 g-3+n}$ embeds $\mathcal{B}$ in $\mathbb{C}^{3 g-3+n}$ as an open ball;

- $C:=\pi^{-1}(y)$ is the most degenerate curve in the pullback $\mathcal{C} \stackrel{\pi}{\rightarrow} \mathcal{B}$ of the universal family over $\mathcal{B}$;

- an étale groupoid representing $\overline{\mathcal{M}}_{g, n}$ trivializes over $\mathcal{B}$ to $\operatorname{Aut}(C) \times \mathcal{B} \rightrightarrows \mathcal{B}$.

Let $\left\{Q_{1}, \ldots, Q_{s}\right\}$ be the set of singular points of $C$ and let $z_{i}$, for $i=1, \ldots, s$, parametrize curves where the singularity $Q_{i}$ subsists. The discriminant locus $\partial \mathcal{B} \subset \mathcal{B}$ of $\pi$ has then equation $z_{1} \cdots z_{S}=0$. Let $U=\mathcal{B} \backslash \partial \mathcal{B}$. The natural morphism $U \rightarrow \mathcal{M}_{g, n}$ induces a homomorphism of fundamental groups:

$$
\phi_{\lambda}: \pi_{1}(U, a) \rightarrow \pi_{1}\left(\mathcal{M}_{g, n}, a\right) .
$$

If we denote as well by $\Gamma^{\lambda}$ the subgroup of $\pi_{1}\left(\mathcal{M}_{g, n}, a\right)$ determined by the étale cover $\mathcal{M}^{\lambda} \rightarrow \mathcal{M}_{g, n}$, a connected component $U^{\lambda}$ of $U \times \overline{\mathcal{M}}_{g, n} \overline{\mathcal{M}}^{\lambda}$ is then determined by the subgroup $\phi_{\lambda}^{-1}\left(\Gamma^{\lambda}\right)$ of the abelian group $\pi_{1}(U, a)$.

Let us make the above description more explicit. Let $s_{1}, \ldots, s_{n}$ be the sections of the universal family over $\overline{\mathcal{M}}_{g, n}$ and define $\left.\mathcal{C}\right|_{U}:=\mathcal{C} \backslash \bigcup_{i=1}^{n} s_{i}(U)$. Let us fix a homeomorphism between the fiber over $a$ of the morphism $\left.\mathcal{C}\right|_{U} \rightarrow U$ and the marking Riemann surface $S_{g, n}$. In more detail, the local monodromy representation

$$
\rho_{U}: \pi_{1}(U, a) \rightarrow \operatorname{Out}\left(\pi_{1}\left(S_{g, n}, \widetilde{a}\right)\right),
$$

associated to the family $\left.\mathcal{C}\right|_{U} \rightarrow U$, is defined as the composition of the natural morphism $\pi_{1}(U) \rightarrow \pi_{1}\left(\mathcal{M}_{g, n}\right)$ with the universal monodromy representation and can be explicitly described as follows.

Since $U$ is homotopic to the $s$-dimensional torus $\left(S^{1}\right)^{s}$, the fundamental group $\pi_{1}(U, a)$ is abelian and freely generated by simple loops $\gamma_{i}$ around the divisors $z_{i}$, for $i=1, \ldots, s$. Such loops can be lifted to disjoint loops $\tilde{\gamma}_{i}$, for $i=1, \ldots, s$, in $S_{g, n}$, whose isotopy classes are uniquely determined and which become isotrivial after specialization to $C$. One can prove that $\gamma_{i}$ is mapped by $\rho_{U}$ exactly in the element 
of $\operatorname{Out}\left(\pi_{1}\left(S_{g, n}, \tilde{a}\right)\right)$ determined by the Dehn twist $\tau_{\tilde{\gamma}_{i}}$ along $\tilde{\gamma}_{i}$, for $i=1, \ldots, s$. In particular, the representation $\rho_{U}$ is faithful.

Let $E_{\Sigma(C)}$ be the free abelian group spanned by the edges of the dual graph $\Sigma(C)$ of the stable curve $C$. The edges of the dual graph correspond to isotopy classes of s.c.c. $\gamma_{e}$ in $S_{g, n}$ which become isotrivial specializing to $C$. The group $E_{\Sigma(C)}$ can then be naturally identified with the free abelian group spanned in $\Gamma_{g, n}$ by the set of Dehn twists $\left\{\tau_{\gamma_{e}}\right\}$. On the other hand, the fundamental group $\pi_{1}(U, a)$ is as well naturally isomorphic to $E_{\Sigma(C)}$. So for a level $\Gamma^{\lambda}$ the subgroup $\phi_{\lambda}^{-1}\left(\Gamma^{\lambda}\right)$ of the fundamental group $\pi_{1}(U, a)$ is then canonically identified with $E_{\Sigma(C)} \cap \Gamma^{\lambda}$.

An almost complete description of local monodromy coefficients for the geometric levels $\Gamma^{w(k, l)}$ is given in [26, Theorem 3.1.3], [6, Proposition 2.8] and [25, Theorem 3.3.3]. Let us collect all their results in a single statement.

Let $N_{\Sigma(C)}$ and $S_{\Sigma(C)}$ be respectively the subgroups of $E_{\Sigma(C)}$ spanned by edges corresponding to nonseparating s.c.c. and by edges corresponding to separating s.c.c.. Let then $S_{\Sigma(C)}^{1}$ be the subgroup of $S_{\Sigma(C)}$ spanned by edges corresponding to separating s.c.c. bounding an unpunctured genus 1 surface. Let instead $P_{\Sigma(C)}$ be the subgroup spanned by elements of the form $e_{1}-e_{2}$, where $\left\{e_{1}, e_{2}\right\}$ corresponds to a cut pair on $C$. Eventually, denote by $P_{\Sigma(C)}^{\text {un }}$ the subgroup of $P_{\Sigma(C)}$ spanned by elements corresponding to cut pairs bounding a surface without punctures. Let, for $m, s$ positive integers, $m_{s}:=m / \operatorname{gcd}(m, s)$.

Theorem 2.2 With the above notation, the kernel of $\rho_{U}^{w(k, l)}$, where $U$ is a neighborhood of $[C] \in \overline{\mathcal{M}}_{g, n}$ as above, is given by:

(1) If $k=2$ :

(2) If $k=3$ :

(3) If $k=4$ and $m$ is odd or $4 \mid m$ : If $k=4$ and $2 \| m:$

(4) If $k \geq 4$ and $\operatorname{gcd}(m, 6)=1$ :

$$
\begin{aligned}
& m N_{\Sigma(C)}+P_{\Sigma(C)}+S_{\Sigma(C)} \\
& m N_{\Sigma(C)}+m_{2} P_{\Sigma(C)}^{\text {un }}+S_{\Sigma(C)} \\
& m N_{\Sigma(C)}+m_{2} S_{\Sigma(C)}+m_{6} S_{\Sigma(C)}^{1} \\
& m N_{\Sigma(C)}+\frac{m}{2} P_{\Sigma(C)}+\frac{m}{2} S_{\Sigma(C)}+\frac{m_{3}}{2} S_{\Sigma(C)}^{1} \\
& m E_{\Sigma(C)}
\end{aligned}
$$

\section{Moduli spaces of stable curves of compact type}

In this section, we begin the study of the moduli space $\widetilde{\mathcal{M}}_{g, n}$ of stable $n$-pointed, genus $g$, complex curves of compact type. We will give various characterization both of its topological fundamental group $\pi_{1}\left(\widetilde{\mathcal{M}}_{g, n}, a\right)$ and of its algebraic fundamental group $\hat{\pi}_{1}\left(\widetilde{\mathcal{M}}_{g, n}, a\right)$ (in the sequel, we will often omit any mention of base points). As 
usual, the profinite completion of a given group $G$ is denoted by $\widehat{G}$. This motivates the previous notation for the algebraic fundamental group of $\widetilde{\mathcal{M}}_{g, n}$.

Since $\partial \widetilde{\mathcal{M}}_{g, n}:=\widetilde{\mathcal{M}}_{g, n} \backslash \mathcal{M}_{g, n}$ is a normal crossing divisor, the embedding $\mathcal{M}_{g, n} \hookrightarrow$ $\widetilde{\mathcal{M}}_{g, n}$ induces an epimorphism $\pi_{1}\left(\mathcal{M}_{g, n}, a\right) \rightarrow \pi_{1}\left(\widetilde{\mathcal{M}}_{g, n}, a\right)$ on fundamental groups, whose kernel is normally generated by small loops around the irreducible components of $\partial \widetilde{\mathcal{M}}_{g, n}$. As we remarked in Section 2, the isomorphism $\Gamma_{g, n} \cong \pi_{1}\left(\mathcal{M}_{g, n}, a\right)$ identifies such loops with Dehn twists along separating s.c.c.. Let us then define

$$
\left.\mathcal{K}_{g, n}:=\left\langle\tau_{\alpha} \in \Gamma_{g, n}\right| \alpha \text { is a separating s.c.c. on } S_{g, n}\right\rangle,
$$

which is also called the Johnson subgroup of $\Gamma_{g, n}$. We then get the following well known characterization of $\pi_{1}\left(\widetilde{\mathcal{M}}_{g, n}, a\right)$, first considered in Hain and Looijenga [9]:

Proposition 3.1 Let $2 g-2+n>0$. The fundamental group of $\widetilde{\mathcal{M}}_{g, n}$ fits in the short exact sequence:

$$
1 \rightarrow \mathcal{K}_{g, n} \rightarrow \Gamma_{g, n} \rightarrow \pi_{1}\left(\widetilde{\mathcal{M}}_{g, n}, a\right) \rightarrow 1
$$

The following definition is then natural:

Definition 3.2 For $2 g-2+n>0$, let $\widetilde{\Gamma}_{g, n}:=\Gamma_{g, n} / \mathcal{K}_{g, n}$ and let us denote by $\widetilde{\Gamma}_{g, n}^{\wedge}$ its profinite completion. The isomorphism $\Gamma_{g, n} \cong \pi_{1}\left(\mathcal{M}_{g, n}, a\right)$ then identifies the groups $\widetilde{\Gamma}_{g, n}$ and $\widetilde{\Gamma}_{g, n}^{\wedge}$ with the topological and the algebraic fundamental group of $\widetilde{\mathcal{M}}_{g, n}$, respectively.

By Theorem 2 in Birman [3], for the case $g=2$, and Theorem 6 in Johnson [13], for the case $g>2$, we know that $\mathcal{K}_{g}$ is the kernel, for $n=0$, of the natural representation

$$
\rho_{W^{3}}: \Gamma_{g, n} \rightarrow \operatorname{Out}\left(\Pi_{g, n} / W^{3} \Pi\right) .
$$

In particular, the fundamental group of $\widetilde{\mathcal{M}}_{g}$ is naturally isomorphic to $\operatorname{Im} \rho_{W^{3}}$. Passing to profinite completions, the above representation induces a continuous representation:

$$
\widehat{\rho}_{W^{3}}: \widehat{\Gamma}_{g, n} \rightarrow \operatorname{Out}\left(\widehat{\Pi}_{g} / W^{3} \widehat{\Pi}\right) .
$$

A natural guess then is that, for $n=0$, the algebraic fundamental group of $\widetilde{\mathcal{M}}_{g, n}$ is isomorphic to $\operatorname{Im} \hat{\rho}_{W^{3}}$. Thanks to a straightforward generalization of Corollary 3.11 in Boggi and Pikaart [6], we will be able to prove that this actually holds for all $n \geq 0$. In its turn, this will yield that the fundamental group of $\widetilde{\mathcal{M}}_{g, n}$ is isomorphic to $\operatorname{Im} \rho_{W^{3}}$, for all $n \geq 0$. Besides more complete results with respect to [6], we will give here more detailed proofs. Let us begin with the following preliminary lemma's. 
Lemma 3.3 Let $f: \mathcal{C} \rightarrow \mathcal{U}$ be a representable, flat, generically smooth morphism, with connected fibers, over an irreducible, reduced, uniformizable complex $D-M$ stack $\mathcal{U}$. Let $\mathcal{C}_{u}$ be the fiber over a point $u \in \mathcal{U}$, and let also $\tilde{u} \in \mathcal{C}_{u}$. There is then an exact sequence of topological fundamental groups:

$$
\pi_{1}\left(\mathcal{C}_{u}, \tilde{u}\right) \rightarrow \pi_{1}(\mathcal{C}, \tilde{u}) \rightarrow \pi_{1}(\mathcal{U}, u) \rightarrow 1 .
$$

Proof Right exactness of the above sequence follows from the fact that $f$ is surjective with connected fibers. In order to prove left exactness, let us consider the universal covers $\tilde{\mathcal{C}}$ and $\tilde{\mathcal{U}}$ of the D-M stacks $\mathcal{C}$ and $\mathcal{U}$, respectively. By hypothesis, such universal covers exist in the category of analytic spaces. Then, the given morphism $f: \mathcal{C} \rightarrow \mathcal{U}$ can be lifted to a morphism of analytic spaces $\tilde{f}: \widetilde{\mathcal{C}} \rightarrow \widetilde{\mathcal{U}}$ and the above sequence is left exact, for a choice of $u \in \mathcal{U}$, if and only if, for any $\tilde{u} \in \tilde{\mathcal{U}}$ lying over $u$, the fiber $\tilde{f}^{-1}(\tilde{u})$ is connected.

Let $\mathcal{U}^{0}$ be the open substack of $\mathcal{U}$ over which $f$ is smooth, $\mathcal{C}^{0}:=f^{-1}\left(\mathcal{U}^{0}\right)$ and $f^{0}:=\left.f\right|_{\mathcal{C}^{0}}$. Let then $\widetilde{\mathcal{C}}^{0}$ and $\widetilde{\mathcal{U}}^{0}$ be the universal covers of $\mathcal{C}$ and $\mathcal{U}$, respectively and let $\tilde{f}^{0}: \widetilde{\mathcal{C}}^{0} \rightarrow \widetilde{\mathcal{U}}^{0}$ be a lift of $f^{0}$ which fits in the commutative diagram:

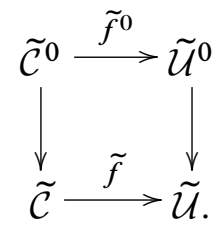

Since $f^{0}$ is a Serre fibration with connected fibers, it is clear that $\widetilde{f}^{0}$ has connected fibers. Therefore, the same holds for the restriction of $\tilde{f}$ to the inverse image of $\mathcal{C}^{0}$ in $\tilde{\mathcal{C}}$, ie all the fibers of $\tilde{f}$ above the inverse image of $\mathcal{U}^{0}$ in $\tilde{\mathcal{U}}$ are connected.

From the flatness of $f$, it follows that $\tilde{f}: \widetilde{\mathcal{C}} \rightarrow \tilde{\mathcal{U}}$ is a flat family of analytic spaces. Moreover, over an open dense subset of $\widetilde{\mathcal{U}}$, the fibers of $\tilde{f}$ are smooth and connected. Any fiber can then, at least locally, be realized as the limit of the smooth connected fibers and so is connected.

Let us denote by $\beta_{0}: \overline{\mathcal{M}}_{g-1, n+2} \rightarrow \overline{\mathcal{M}}_{g, n}$ the natural pinching morphism whose image is the locus whose generic point parametrizes singular irreducible curves. An étale cover $\widetilde{\mathcal{M}}^{\lambda}$ of $\widetilde{\mathcal{M}}_{g, n}$ compactifies to an étale cover $\overline{\mathcal{M}}^{\lambda} \rightarrow \overline{\mathcal{M}}_{g, n}$ whose branch locus is contained in the image of $\beta_{0}$.

Lemma 3.4 (i) Let $\overline{\mathcal{M}}^{\lambda} \rightarrow \overline{\mathcal{M}}_{g, n}$ be a level structure whose branch locus is contained in the boundary divisor $\beta_{0}\left(\overline{\mathcal{M}}_{g-1, n+2}\right)$. Denote by $p: \Gamma_{g, n} \rightarrow \Gamma_{g, n-1}$ the epimorphism induced filling in $P_{n}$ on $S_{g, n}$ and let $\Gamma^{p(\lambda)}:=p\left(\Gamma^{\lambda}\right)$. Then $\pi_{1}\left(\overline{\mathcal{M}}_{g, n}^{\lambda}\right)=\pi_{1}\left(\overline{\mathcal{M}}_{g, n-1}^{p(\lambda)}\right)$. 
(ii) Let $\Gamma^{\lambda_{1}} \leq \Gamma^{\lambda_{2}}$ be two levels whose associated level structures satisfy the hypothesis in the above item. If the natural morphism $\overline{\mathcal{M}}_{g, n}^{\lambda_{1}} \rightarrow \overline{\mathcal{M}}_{g, n}^{\lambda_{2}}$ is étale and, with the same notation as above, $p\left(\Gamma^{\lambda_{1}}\right)=p\left(\Gamma^{\lambda_{2}}\right)$, then $\Gamma^{\lambda_{1}}=\Gamma^{\lambda_{2}}$.

Proof Item (ii) follows directly from (i). So let us prove (i). We claim that the natural morphism $\phi: \mathcal{M}_{g, n}^{\lambda} \rightarrow \mathcal{M}_{g, n-1}^{p(\lambda)}$ is a fibration in smooth curves. There is a factorization

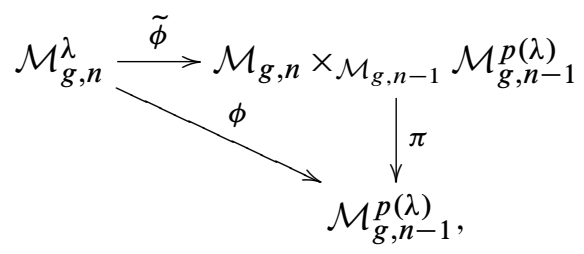

with $\widetilde{\phi}$ étale and $\pi$ smooth. Thus $\phi$ is also smooth. In the Stein factorization of $\phi$

$$
\mathcal{M}_{g, n}^{\lambda} \stackrel{\phi^{\prime}}{\longrightarrow} Y \stackrel{f}{\longrightarrow} \mathcal{M}_{g, n-1}^{p(\lambda)},
$$

the morphism $\phi^{\prime}$ has connected fibers and $f$ is finite. Therefore $f$ is étale. By definition of $\Gamma^{p(\lambda)}$, the map induced on fundamental groups by $\phi$ is surjective. So the same is true for the morphism $f$ which then is an isomorphism. Hence $\phi=\phi^{\prime}$. In particular, since the morphism $\phi: \overline{\mathcal{M}}_{g, n}^{\lambda} \rightarrow \overline{\mathcal{M}}_{g, n-1}^{p(\lambda)}$ is also log-smooth, it is a flat family of semistable curves. Let $S$ be a fiber of $\phi$ lying above a fiber of $\overline{\mathcal{M}}_{g, n} \rightarrow$ $\overline{\mathcal{M}}_{g, n-1}$ which is a tree of $g$ elliptic curves. By the assumptions made on the branch locus of $\overline{\mathcal{M}}_{g, n}^{\lambda} \rightarrow \overline{\mathcal{M}}_{g, n}$, the induced cover $S \rightarrow C$ is étale. Therefore $S$ is a tree of elliptic curves as well. Note that for any s.c.c. $\alpha$ on one of the components of $S$ there is a degeneration of $S$ in the family $\overline{\mathcal{M}}_{g, n}^{\lambda} \rightarrow \overline{\mathcal{M}}_{g, n-1}^{p(\lambda)}$, induced by a degeneration of $C$ in the family $\overline{\mathcal{M}}_{g, n} \rightarrow \overline{\mathcal{M}}_{g, n-1}$, in which $\alpha$ is a vanishing loop.

From the exact sequence of groups

$$
\pi_{1}(S) \rightarrow \pi_{1}\left(\overline{\mathcal{M}}_{g, n}^{\lambda}\right) \stackrel{\phi}{\rightarrow} \pi_{1}\left(\overline{\mathcal{M}}_{g, n-1}^{p(\lambda)}\right) \rightarrow 1,
$$

given by Lemma 3.3 , it follows that $\pi_{1}\left(\overline{\mathcal{M}}_{g, n}^{\lambda}\right)=\pi_{1}\left(\overline{\mathcal{M}}_{g, n-1}^{p(\lambda)}\right)$.

In the previous section, we defined the Galois level $\Gamma^{w(k, m)}$ as the kernel of the natural representation $\rho_{W^{k, m}}: \Gamma_{g, n} \rightarrow \operatorname{Out}\left(\Pi_{g, n} / W^{k, m} \Pi\right)$.

The group $\mathcal{K}_{g, n}(m)$ is defined, for $2 g-2+n>0$, to be the normal subgroup of $\Gamma_{g, n}$ generated by Dehn twists along separating s.c.c., $m$-th powers of Dehn twists along nonseparating s.c.c. and $m_{2}$-th powers of bounding pair maps $\tau_{\gamma_{1}}^{-1} \tau_{\gamma_{2}}$, for all cut pairs $\gamma_{1}, \gamma_{2}$ bounding an unmarked subsurface of $S_{g, n}$ (as usual, we let $m_{2}:=m / \operatorname{gcd}(2, m)$ ). 
Proposition 3.5 Let $g \geq 2$. Then, $\Gamma_{g}^{w(3, m)}=\mathcal{K}_{g}(m)$.

Proof For $g=2$, the proposition is just Proposition 3.2 in [6]. The case $g \geq 3$ and $m$ odd was already treated in Proposition 3.4 in [6]. Here, we reproduce that proof with the modifications needed in order to make it work also for $m$ even.

From Theorem 2.2, it follows that $\mathcal{K}_{g}(m) \leq \Gamma_{g}^{w(3, m)}$. It then holds $\mathcal{K}_{g}(m) \cdot \mathcal{T}_{g}=$ $\Gamma_{g}^{w(3, m)} \cdot \mathcal{T}_{g}$, since, by Proposition 3.4 in [6], both groups equal $\Gamma(m)$. There is then a commutative diagram with exact rows:

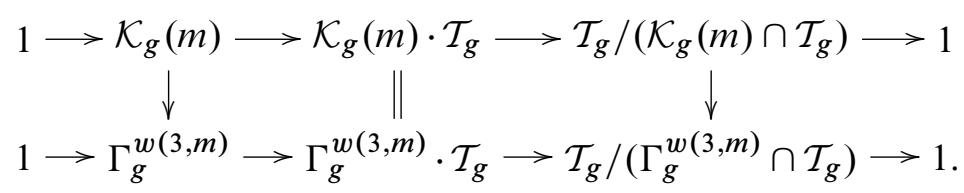

Therefore, in order to prove the proposition, it is enough to show that $\mathcal{K}_{g}(m) \cap \mathcal{T}_{g}=$ $\Gamma_{g}^{w(3, m)} \cap \mathcal{T}_{g}$ and then that:

$$
\left(\mathcal{K}_{g}(m) \cap \mathcal{T}_{g}\right) / \mathcal{K}_{g}=\left(\Gamma_{g}^{w(3, m)} \cap \mathcal{T}_{g}\right) / \mathcal{K}_{g}
$$

The advantage of considering the latter identity is that it can be checked inside a torsion free abelian group. More precisely, there is a natural representation, Johnson's homomorphism (defined in [11]):

$$
j_{0}: \mathcal{T}_{g} \rightarrow \wedge^{3} H_{1}\left(S_{g}, \mathbb{Z}\right) /\left(\left[S_{g}\right] \wedge H_{1}\left(S_{g}, \mathbb{Z}\right)\right),
$$

where $\left[S_{g}\right] \in \wedge^{2} H_{1}\left(S_{g}, \mathbb{Z}\right)$ is the fundamental class of $S_{g}$. The right-hand side is a free $\mathbb{Z}$-module of rank $\left(\begin{array}{c}2 g \\ 3\end{array}\right)-2 g$ and $\operatorname{ker} j_{0}=\mathcal{K}_{g}$.

Corollary 6.4 in [26] implies that the image of the group $\pi_{1}\left(S_{g}\right)^{[3], m}$ in the free abelian group $\pi_{1}\left(S_{g}\right)^{[2]} / \pi_{1}\left(S_{g}\right)^{[3]}$ is the subgroup of $m_{2}$-th powers, ie

$$
\pi_{1}\left(S_{g}\right)^{[3], m} / \pi_{1}\left(S_{g}\right)^{[3]} \cong m_{2} \cdot \wedge^{2} H_{1}\left(S_{g}, \mathbb{Z}\right) /\left[S_{g}\right] .
$$

Sp the image of $H_{1}\left(S_{g}, \mathbb{Z}\right) \otimes \pi_{1}\left(S_{g}\right)^{[3], m}$ inside $\wedge^{3} H_{1}\left(S_{g}, \mathbb{Z}\right) /\left(\left[S_{g}\right] \wedge H_{1}\left(S_{g}, \mathbb{Z}\right)\right)$ equals the submodule $m_{2} \cdot \wedge{ }^{3} H_{1}\left(S_{g}, \mathbb{Z}\right) /\left(\left[S_{g}\right] \wedge H_{1}\left(S_{g}, \mathbb{Z}\right)\right)$.

By the computations which are carried out in [11], we know that the group $\mathcal{T}_{g} / \mathcal{K}_{g}$ embeds as a primitive submodule, with a basis given by bounding pair maps, in the free $\mathbb{Z}$-module $\wedge{ }^{3} H_{1}\left(S_{g}, \mathbb{Z}\right) /\left(\left[S_{g}\right] \wedge H_{1}\left(S_{g}, \mathbb{Z}\right)\right)$. It then follows that an element of $\mathcal{T}_{g} / \mathcal{K}_{g}$ belongs to the submodule of $m_{2}$-th powers if and only if it can be represented as a product of $m_{2}$-th powers of bounding pair maps. This immediately yields the identity $(*)$, thus completing the proof of the proposition.

The second item of the following theorem is a generalization of Theorem 3.5 in [6]: 
Theorem 3.6 (i) Let $n>0$. For all $m \geq 2$, there are natural isomorphism $\pi_{1}\left(\overline{\mathcal{M}}_{1, n}^{w(3, m)}\right) \cong \pi_{1}\left(\overline{\mathcal{M}}_{1,1}^{(m)}\right)$. Therefore, $\bar{\Gamma}_{1, n}^{w(3, m)} / \mathcal{K}_{1, n}(m) \cong \pi_{1}\left(\overline{\mathcal{M}}_{1,1}^{(m)}\right)$.

(ii) Let $g \geq 2$ and $n \geq 0$. For $m \geq 2$, the level structure $\overline{\mathcal{M}}^{w(3, m)}$ over $\overline{\mathcal{M}}_{g, n}$ is simply connected. Therefore, $\Gamma^{w(3, m)}=\mathcal{K}_{g, n}(m)$.

Proof We have Out ${ }^{+}\left(\Pi_{1,1} / W^{3, m} \Pi\right)=\mathrm{SL}_{2}(\mathbb{Z} / m)$. Therefore, for $g=1$ and $n=1$, we have $\Gamma^{w(3, m)}=\Gamma(m) \cong \pi_{1}\left(\mathcal{M}_{1,1}^{(m)}\right)$. The statement of the theorem, for $g=1$ and $n=1$, then follows since the generators of $\mathcal{K}_{1,1}(m)$ correspond, in the fundamental group of $\mathcal{M}_{1,1}^{(m)}$, to small loops around the punctures.

For $g \geq 2$ and $n=0,1$, from Proposition 3.5 and simple topological arguments, like the ones given in the proof of Proposition 3.3 in [6], it follows that the level structures $\overline{\mathcal{M}}_{g, n}^{w(3, m)}$ are simply connected.

For $n \geq 2$, we proceed by induction on $n$. Let us then assume that the statement of the theorem has been proved up to $n-1$ and let us prove it for $n$. By Lemma 3.4 and Theorem 2.2, it is enough to prove, with the same notation of the lemma, the identity:

$$
p\left(\Gamma_{g, n}^{w(3, m)}\right)=\Gamma_{g, n-1}^{w(3, m)} .
$$

The inclusion $p\left(\Gamma_{g, n}^{w(3, m)}\right) \leq \Gamma_{g, n-1}^{w(3, m)}$ is trivial, since the epimorphism $\Pi_{g, n} \rightarrow \Pi_{g, n-1}$ induces an epimorphism $\Pi_{g, n} / W^{3, m} \Pi \rightarrow \Pi_{g, n-1} / W^{3, m} \Pi$.

For $g \geq 2$, the reverse inclusion follows, from the fact that, by inductive hypothesis:

$$
\Gamma_{g, n-1}^{w(3, m)}=\mathcal{K}_{g, n-1}(m)=p\left(\mathcal{K}_{g, n}(m)\right) \leq p\left(\Gamma_{g, n}^{w(3, m)}\right) .
$$

For $g=1$ and all $n \geq 1$, there is a series of natural epimorphism:

$$
\Gamma_{1, n}^{w(3, m)} / \mathcal{K}_{1, n}(m) \rightarrow \pi_{1}\left(\mathcal{M}_{1,1}^{(m)}\right) \rightarrow \pi_{1}\left(\overline{\mathcal{M}}_{1,1}^{(m)}\right) .
$$

Since, by inductive hypothesis, $\Gamma_{1, n-1}^{w(3, m)} / \mathcal{K}_{1, n-1}(m) \cong \pi_{1}\left(\overline{\mathcal{M}}_{1,1}^{(m)}\right)$ and, moreover, $p\left(\mathcal{K}_{1, n}(m)\right)=\mathcal{K}_{1, n-1}(m)$, it follows that the natural homomorphism $p: \Gamma_{1, n}^{w(3, m)} \rightarrow$ $\Gamma_{1, n-1}^{w(3, m)}$ is surjective.

In order to complete the proof of the theorem, let us just remark that, again by simple topological arguments, like those in the proof of Corollary 3.11 in [6], the assertion about the simply connectedness of the level structures $\overline{\mathcal{M}}^{w(3, m)}$ implies the assertion about the generators for the corresponding levels.

Of course, the profinite group $\widetilde{\Gamma}^{\wedge} \cong \widehat{\Gamma / \mathcal{K}}$ is naturally isomorphic to $\lim _{m>0} \Gamma / \mathcal{K}(m)$. From Theorem 3.6, we then have the following: 
Theorem 3.7 Let $g \geq 2$ and $n \geq 0$. The algebraic fundamental group of $\widetilde{\mathcal{M}}_{g, n}$ is naturally isomorphic to $\lim _{m>0} \Gamma_{g, n} / \Gamma^{w(3, m)}$, ie to the image of the representation $\widehat{\rho}_{W^{3}}$.

Remark 3.8 For $g=1$ and $n=1$, from Theorem 3.6, it follows that

$$
\lim _{m>0} \Gamma_{1,1} / \Gamma^{w(3, m)} \cong \mathrm{SL}_{2}(\widehat{\mathbb{Z}})
$$

which is not the profinite completion of $\widetilde{\Gamma}_{1,1} \cong \mathrm{SL}_{2}(\mathbb{Z})$. In fact, according to Theorem 8.8.1 in [27], the kernel of the natural homomorphism $\widehat{\mathrm{SL}_{2}(\mathbb{Z})} \rightarrow \mathrm{SL}_{2}(\widehat{\mathbb{Z}})$ is a free profinite group of countably infinite rank, which we denote by $\widehat{F}_{\infty}$.

Let $2 g-2+n>0$, in general, we have $\widetilde{\Gamma}_{g, n}^{\wedge} \cong \widehat{\Gamma}_{g, n} / \overline{\mathcal{K}}_{g, n}$, where $\widehat{\Gamma}_{g, n}$ denotes the profinite completion of the Teichmüller group $\Gamma_{g, n}$ and $\overline{\mathcal{K}}_{g, n}$ the closure of the group $\mathcal{K}_{g, n}$ inside $\widehat{\Gamma}_{g, n}$. Of course, $\overline{\mathcal{K}}_{g, n} \leq \operatorname{ker} \widehat{\rho}_{W^{3}}$, therefore the representation $\hat{\rho}_{W^{3}}$ induces a natural homomorphism, which we denote in the same way:

$$
\hat{\rho}_{W^{3}}: \widetilde{\Gamma}_{g, n}^{\wedge} \rightarrow \operatorname{Out}\left(\widehat{\Pi}_{g, n} / W^{3} \widehat{\Pi}\right) .
$$

By Theorem 3.7, $\hat{\rho}_{W^{3}}$ is injective for $g \geq 2$. For $g=1$, the following result holds:

Theorem 3.9 For $n \geq 1$, the kernel of the natural representation

$$
\widetilde{\Gamma}_{1, n}^{\wedge} \rightarrow \operatorname{Out}\left(\widehat{\Pi}_{1, n} / W^{3} \widehat{\Pi}\right)
$$

is naturally isomorphic to the congruence kernel $\widehat{F}_{\infty}$.

Proof Let us denote by $\overline{\mathcal{K}}_{1, n}(m)$ the closure of the group $\mathcal{K}_{1, n}(m)$ in the profinite group $\widehat{\Gamma}_{1, n}$. By Theorem 3.6, there is a natural isomorphism $\widehat{\Gamma}_{1, n}^{w(3, m)} / \overline{\mathcal{K}}_{1, n}(m) \cong$ $\hat{\pi}_{1}\left(\overline{\mathcal{M}}_{1,1}^{(m)}\right)$. Let us now observe that, identifying the fundamental group of the level structure $\mathcal{M}_{1,1}^{(m)}$ with the level $\Gamma(m)$, there is a natural isomorphism

$$
\left.\pi_{1}\left(\overline{\mathcal{M}}_{1,1}^{(m)}\right) \cong \Gamma(m) /\left\langle\tau_{\gamma}^{m}\right| \gamma \text { a s.c.c. on } S_{1,1}\right\rangle .
$$

Therefore, there is a series of natural isomorphisms:

$$
\begin{aligned}
\lim _{m>0}\left(\widehat{\Gamma}_{1, n}^{w(3, m)} / \overline{\mathcal{K}}_{1, n}(m)\right) & \cong \lim _{m>0} \widehat{\pi}_{1}\left(\overline{\mathcal{M}}_{1,1}^{(m)}\right) \\
& \cong \lim _{m>0}\left(\widehat{\Gamma}(m) / \overline{\left.\left\langle\tau_{\gamma}^{m}\right| \gamma \text { a s.c.c. on } S_{1,1}\right\rangle}\right)={\underset{\lim }{m>0}}_{\overleftarrow{\Gamma}(m)=\widehat{F}_{\infty} .}
\end{aligned}
$$


The theorem then follows taking the inverse limit on $m$ of the exact sequences of profinite groups:

$$
1 \rightarrow \widehat{\Gamma}_{1, n}^{w(3, m)} / \overline{\mathcal{K}}_{1, n}(m) \rightarrow \widetilde{\Gamma}_{1, n}^{\wedge} \rightarrow \operatorname{Out}\left(\widehat{\Pi}_{1, n} / W^{3, m} \widehat{\Pi}\right) .
$$

An easy consequence of Theorem 3.6 is also:

Proposition 3.10 For $g \geq 2$ and $n \geq 0$, let $\widetilde{\mathcal{M}}^{\lambda}$ be a finite connected étale cover of $\widetilde{\mathcal{M}}_{g, n}$, then its compactification $\overline{\mathcal{M}}^{\lambda}$ over $\overline{\mathcal{M}}_{g, n}$ has finite fundamental group.

Proof Let $m$ be the 1.c.m. of the ramification indices of the cover $\overline{\mathcal{M}}^{\lambda} \rightarrow \overline{\mathcal{M}}_{g, n}$ over $\beta_{0}\left(\overline{\mathcal{M}}_{g-1, n+2}\right)$ and let $\bar{X}$ be the universal cover of $\overline{\mathcal{M}}^{\lambda}$ and $X$ the inverse image of $\mathcal{M}_{g, n}$ in $\bar{X}$. Then it is clear that $\Gamma_{g, n} \geq \pi_{1}(X) \geq \mathcal{K}_{g, n}(2 m)$. Therefore, $\pi_{1}(X)$ is a finite index subgroup of $\Gamma_{g, n}$ and $\bar{X} \rightarrow \overline{\mathcal{M}}^{\lambda}$ a finite cover.

Corollary 3.6 in [6] can now be substantially improved as well the result by Hain in [8] about the vanishing of the first cohomology group of levels containing the Torelli group:

Corollary 3.11 Let $\Gamma^{\lambda}$ be a level in $\Gamma_{g, n}$ containing $\mathcal{K}_{g, n}$.

(i) For $g \geq 2$ and $n \geq 0$, we have $H^{1}\left(\overline{\mathcal{M}}^{\lambda}, \mathbb{Z}\right)=0$.

(ii) For $g \geq 3$ and $n=0$, we have $H^{1}\left(\Gamma^{\lambda}, \mathbb{Z}\right)=0$.

Proof The first statement immediately follows from Proposition 3.10. For the second one, see the proof of Corollary 3.6 in Boggi and Pikaart [6].

The profinite group $\widehat{\Pi}_{g, n} / W^{3} \widehat{\Pi}$ is nilpotent. Therefore, there is a natural isomorphism

$$
\widehat{\Pi}_{g, n} / W^{3} \widehat{\Pi} \cong \prod_{\ell \text { prime }} \Pi_{g, n}^{(\ell)} / W^{3} \Pi^{(\ell)},
$$

where, for a given prime $\ell>0$, we denote by $\Pi_{g, n}^{(\ell)}$ the pro- $\ell$ completion of $\Pi_{g, n}$.

Let us define the group $\widetilde{\Gamma}_{g, n}^{(\ell)}$, for a given prime $\ell>0$ and $g \geq 2$, to be the closure of the image of $\Gamma_{g, n}$ inside the virtual pro- $\ell$ group $\operatorname{Out}\left(\Pi_{g, n}^{(\ell)} / W^{3} \Pi^{(\ell)}\right)$. We then have for $g \geq 2$ :

$$
\widetilde{\Gamma}_{g, n}^{\wedge} \cong \prod_{\ell \text { prime }} \widetilde{\Gamma}_{g, n}^{(\ell)}
$$

In Corollary 7.2 of Hain and Looijenga [9], the fundamental groups of $\widetilde{\mathcal{M}}_{g}$ and $\widetilde{\mathcal{M}}_{g, 1}$ are explicitly described. Let us recall this description. Let us denote by $H$ the first integral homology group of the compact surface $S_{g}$, obtained filling in the punctures 
on $S_{g, n}$, and let then $\omega \in \wedge^{2} H$ be the fundamental class of $S_{g}$. According to some results of Johnson $\left[11 ; 12 ; 13\right.$, for $n=0,1$, the Johnson's group $\mathcal{K}_{g, n}$ is the kernel of some naturally defined surjective linear representations:

$$
j_{0}: \mathcal{T}_{g} \rightarrow \wedge^{3} H /(\omega \wedge H) \quad \text { and } \quad j_{1}: \mathcal{T}_{g, 1} \rightarrow \wedge^{3} H,
$$

called Johnson's homomorphisms. For $n=0$, by Johnson's definition, ker $j_{0}=\operatorname{ker} \rho_{W^{3}}$. Therefore, the fundamental groups of $\widetilde{\mathcal{M}}_{g}$ and $\widetilde{\mathcal{M}}_{g, 1}$ fit in the short exact sequences:

$$
\begin{aligned}
& 1 \rightarrow \wedge^{3} H /(\omega \wedge H) \rightarrow \widetilde{\Gamma}_{g} \rightarrow \operatorname{Sp}(H) \rightarrow 1 \\
& 1 \rightarrow \wedge^{3} H \rightarrow \widetilde{\Gamma}_{g, 1} \rightarrow \operatorname{Sp}(H) \rightarrow 1 .
\end{aligned}
$$

Let $\hat{H} \cong \widehat{\mathbb{Z}}^{2 g}$ be the profinite completion of $H$. By the congruence subgroup property for symplectic groups [2], the profinite group $\operatorname{Sp}(\hat{H}) \cong \operatorname{Sp}_{2 g}(\hat{\mathbb{Z}})$, for $g \geq 2$, is the profinite completion of $\operatorname{Sp}(H)$. Moreover, from item (ii) of Theorem 3.6, it follows that the images of the levels $\Gamma_{g, n}^{w(3, m)}$ in $\widetilde{\Gamma}_{g, n}$, for $m \geq 0, g \geq 2$ and $n=0,1$, cut a fundamental system of neighborhoods of the identity, for the profinite topology, on the subgroups $\wedge^{3} H /(\omega \wedge H)$ and $\wedge^{3} H$, for $n=0,1$, respectively. Therefore, the algebraic fundamental groups of $\widetilde{\mathcal{M}}_{g}$ and $\widetilde{\mathcal{M}}_{g, 1}$, for $g \geq 2$, are described by the short exact sequences:

$$
\begin{aligned}
& 1 \rightarrow \wedge^{3} \hat{H} /(\omega \wedge \hat{H}) \rightarrow \widetilde{\Gamma}_{g}^{\wedge} \rightarrow \operatorname{Sp}(\hat{H}) \rightarrow 1 \\
& 1 \rightarrow \wedge^{3} \hat{H} \rightarrow \widetilde{\Gamma}_{g, 1}^{\wedge} \rightarrow \operatorname{Sp}(\hat{H}) \rightarrow 1 .
\end{aligned}
$$

More in general, the topological and algebraic fundamental groups of $\widetilde{\mathcal{M}}_{g, n+1}$ can be described as abelian extensions of the topological and algebraic fundamental groups of $\widetilde{\mathcal{M}}_{g, n}$ for all $n \geq 0$ :

Theorem 3.12 For $2 g-2+n>0$, there are the following natural short exact sequences, which, if $n \geq 1$, admit $n$ natural splittings:

$$
\begin{gathered}
1 \rightarrow H \rightarrow \widetilde{\Gamma}_{g, n+1} \rightarrow \widetilde{\Gamma}_{g, n} \rightarrow 1, \\
1 \rightarrow \widehat{H} \rightarrow \widetilde{\Gamma}_{g, n+1}^{\wedge} \rightarrow \widetilde{\Gamma}_{g, n}^{\wedge} \rightarrow 1 .
\end{gathered}
$$

Similarly, for $g \geq 2$ and a prime $\ell>0$, there is a natural short exact sequence, which, if $n \geq 1$, admits $n$ natural splittings:

$$
1 \rightarrow H^{(\ell)} \rightarrow \widetilde{\Gamma}_{g, n+1}^{(\ell)} \rightarrow \widetilde{\Gamma}_{g, n}^{(\ell)} \rightarrow 1,
$$

where $H^{(\ell)}$ denotes the pro- $\ell$ completion of $H$. 
Remark 3.13 As it was pointed out by the referee, the first two sequences do not split, when $n=0$ and $g \geq 3$, and the third does not split when $n=0, g \geq 3$ and $\ell \mid g-1$.

Proof The morphism $\widetilde{\mathcal{M}}_{g, n+1} \rightarrow \widetilde{\mathcal{M}}_{g, n}$ is an $n$-pointed, genus $g$ stable curve. Let $[C] \in \widetilde{\mathcal{M}}_{g, n}$ be such that the curve $C$ is smooth. By Lemma 3.3, there is an exact sequence:

$$
\pi_{1}(C) \rightarrow \widetilde{\Gamma}_{g, n+1} \rightarrow \widetilde{\Gamma}_{g, n} \rightarrow 1 .
$$

Let us show that the homomorphism $\pi_{1}(C) \rightarrow \widetilde{\Gamma}_{g, n+1}$ factors through the abelianization $H_{1}(C)$ of $\pi_{1}(C)$. For $g=1$, this is obvious since $\pi_{1}(C) \cong H_{1}(C)$. For $g \geq 2$, let us fix a homeomorphism of the curve $C$ with the reference genus $g$ compact Riemann surface $S_{g}$. There is a natural commutative diagram with exact rows:

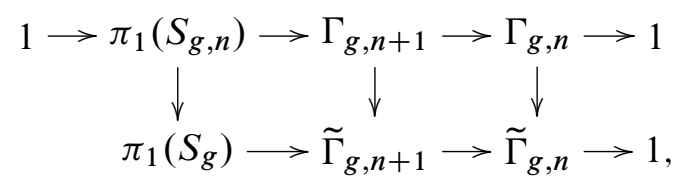

where the vertical arrow $\pi_{1}\left(S_{g, n}\right) \rightarrow \pi_{1}\left(S_{g}\right)$ is the epimorphism induced by the inclusion $S_{g, n} \subset S_{g}$. Let $\gamma$ be a separating s.c.c. on $S_{g, n}$ bounding a disc containing all the punctures of $S_{g, n}$. Let then $S^{\prime}$ be the genus $g$ subsurface of $S_{g, n}$ with boundary $\gamma$ and let $\Gamma\left(S^{\prime}\right)$ be the mapping class group of $S^{\prime}$. Fixing the base-point on $\gamma$, the fundamental group of $S^{\prime}$ is identified with a subgroup of $\Gamma\left(S^{\prime}\right)$. Moreover, there is a natural monomorphism $\Gamma\left(S^{\prime}\right) \hookrightarrow \Gamma_{g, n+1}$ which is compatible with the monomorphism $\pi_{1}\left(S^{\prime}\right) \hookrightarrow \pi_{1}\left(S_{g, n}\right)$ induced by the inclusion $S^{\prime} \subset S_{g, n}$. By Theorem 2 in [3] and Theorem 6 in [13], the quotient of the Torelli subgroup $\mathcal{T}\left(S^{\prime}\right)$ of $\Gamma\left(S^{\prime}\right)$ by the normal subgroup generated by the twists along separating s.c.c. is abelian. In particular, the image of $\mathcal{T}\left(S^{\prime}\right)$ in $\widetilde{\Gamma}_{g, n+1}$ is abelian. Since the homomorphism $\pi_{1}\left(S^{\prime}\right) \rightarrow \pi_{1}\left(S_{g}\right)$ induced by the inclusion $S^{\prime} \subset S_{g}$ is an epimorphism and $\pi_{1}\left(S^{\prime}\right)$ is contained in $\mathcal{T}\left(S^{\prime}\right)$, it follows that the image of $\pi_{1}\left(S_{g}\right)$ in $\widetilde{\Gamma}_{g, n+1}$ is abelian. In order to see that this image is actually the abelianization $H_{1}\left(S_{g}, \mathbb{Z}\right)$, let us observe what follows.

The relative Jacobian $\mathcal{J}_{\tilde{\mathcal{M}}_{g, n+1} / \tilde{\mathcal{M}}_{g, n}}$, parameterizing relative Cartier divisors of degree 0 , is an abelian variety of rank $2 g$ over $\widetilde{\mathcal{M}}_{g, n}$. In particular, it is a Serre fibration endowed with the natural section defined assigning the zero point in the fiber to each point of the base. Therefore, the associated long exact homotopy sequence splits and gives a split short exact sequence:

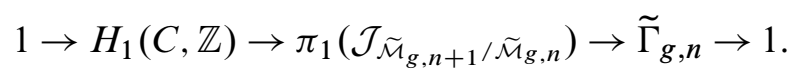

Let us remark that the existence of the splitting implies that the above short exact sequence remains exact passing to profinite completions. 
Let $j: \widetilde{\mathcal{M}}_{g, n+1} \rightarrow \mathcal{J}_{\widetilde{\mathcal{M}}_{g, n+1} / \widetilde{\mathcal{M}}_{g, n}}$ be the map defined assigning to the point of $\widetilde{\mathcal{M}}_{g, n+1}$ parametrizing the pointed stable curve $\left(C, P_{1}, \ldots, P_{n+1}\right)$, the class $(2 g-2) P_{n+1}-\kappa_{C}$ in $\operatorname{Pic}^{0}(C)$, where, by $\kappa_{C}$, we denote the Cartier divisor associated to the dualizing sheaf of the proper curve $C$. This map induces, on fundamental groups, the homomorphism $j_{*}: \widetilde{\Gamma}_{g, n+1} \rightarrow \pi_{1}\left(\mathcal{J}_{\widetilde{\mathcal{M}}_{g, n+1} / \widetilde{\mathcal{M}}_{g, n}}\right)$ which fits in the commutative diagram with exact rows:

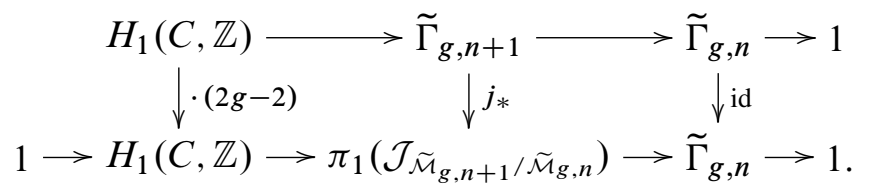

Now, the left hand vertical map is multiplication by $2 g-2$ while the right hand vertical map is the identity. Therefore, exactness of the bottom line implies that the upper line is a short exact sequence as well. The same argument with topological fundamental groups replaced by the algebraic ones implies that, for $2 g-2+n>0$, there is also a short exact sequence:

$$
1 \rightarrow \widehat{H} \rightarrow \widetilde{\Gamma}_{g, n+1}^{\wedge} \rightarrow \widetilde{\Gamma}_{g, n}^{\wedge} \rightarrow 1 .
$$

The last exact sequence in the statement of Theorem 3.12 follows from the very definition of the groups $\widetilde{\Gamma}_{g, n}^{(\ell)}$, for $g \geq 2, n \geq 0$ and a prime $\ell>0$.

Let us conclude observing that, for $n \geq 1$, the tautological sections $s_{i}: \widetilde{\mathcal{M}}_{g, n} \rightarrow$ $\widetilde{\mathcal{M}}_{g, n+1}$, for $i=1, \ldots, n$, of the universal curve $\widetilde{\mathcal{M}}_{g, n+1} \rightarrow \widetilde{\mathcal{M}}_{g, n}$ induce $n$ natural splittings of all the short exact sequences in the statement of Theorem 3.12.

By Theorem 3.12 and the remarks preceding its statement, in particular, the following holds:

Corollary 3.14 Let $2 g-2+n>0$. The group $\widetilde{\Gamma}_{g, n}$ is residually finite.

We can now give a characterization of Johnson's subgroup $\mathcal{K}_{g, n}$ for all $n \geq 0$ :

Theorem 3.15 Let $2 g-2+n>0$. The kernel of $\rho_{W^{3}}: \Gamma_{g, n} \rightarrow \operatorname{Out}\left(\Pi_{g, n} / W^{3} \Pi\right)$ is generated by Dehn twists along separating s.c.c., ie $\operatorname{ker} \rho_{W^{3}}=\mathcal{K}_{g, n}$.

Proof The case of genus 0 is trivial, since $\operatorname{ker} \rho_{W^{3}} \geq \mathcal{K}_{0, n}=\Gamma_{0, n}$.

On the contrary, the case of genus 1 is quite tricky. Let $\widetilde{\Gamma}_{1, n}^{\diamond}:=\widetilde{\Gamma}_{1, n}^{\wedge} / \widehat{F}_{\infty}$, for all $n \geq 1$. By Theorem 3.12 and Theorem 3.9, for all $n \geq 1$, there is a short exact sequence:

$$
1 \rightarrow \widehat{\mathbb{Z}} \oplus \widehat{\mathbb{Z}} \rightarrow \widetilde{\Gamma}_{1, n+1}^{\diamond} \rightarrow \widetilde{\Gamma}_{1, n}^{\diamond} \rightarrow 1 \text {. }
$$


Since $\widetilde{\Gamma}_{1,1}^{\diamond} \cong \mathrm{SL}_{2}(\widehat{\mathbb{Z}})$ and the natural morphism $\mathrm{SL}_{2}(\mathbb{Z}) \rightarrow \mathrm{SL}_{2}(\widehat{\mathbb{Z}})$ is injective, the above short exact sequence and a simple induction argument yield that the natural morphism $\widetilde{\Gamma}_{1, n} \rightarrow \widetilde{\Gamma}_{1, n}^{\diamond}$ is injective for all $n \geq 1$. Then from Theorem 3.9, the natural morphism $\widetilde{\Gamma}_{1, n} \rightarrow \operatorname{Out}\left(\Pi_{1, n} / W^{3} \Pi\right)$ is also injective, which is equivalent to $\operatorname{ker} \rho_{W^{3}}=$ $\mathcal{K}_{1, n}$.

For $g \geq 2$, by Corollary 3.14 and Theorem 3.7, the representation $\rho_{W^{3}}$ induces a monomorphism $\widetilde{\Gamma}_{g, n} \hookrightarrow \operatorname{Out}\left(\widehat{\Pi}_{g, n} / W^{3} \widehat{\Pi}\right)$ which factors through the natural homomorphism $\widetilde{\Gamma}_{g, n} \rightarrow \operatorname{Out}\left(\Pi_{g, n} / W^{3} \Pi\right)$, which then is injective, ie $\operatorname{ker} \rho_{W^{3}}=\mathcal{K}_{g, n}$.

Corollary 3.16 Let $2 g-2+n>0$. The topological fundamental group of $\widetilde{\mathcal{M}}_{g, n}$ is naturally isomorphic to the image of the representation $\rho_{W^{3}}: \Gamma_{g, n} \rightarrow \operatorname{Out}\left(\Pi_{g, n} / W^{3} \Pi\right)$.

It is not hard to extend Johnson's construction of the linear representation $j_{0}$ in [11] to the $n$-pointed case. Let us consider the central abelian extension:

$$
1 \rightarrow W^{2} \Pi / W^{3} \Pi \rightarrow \Pi_{g, n} / W^{3} \Pi \rightarrow H \rightarrow 1 .
$$

Let $f$ be an automorphism of $\Pi_{g, n}$ inducing the identity on $H$, then the assignment $\gamma \mapsto f(\gamma) \cdot \gamma^{-1}$, for $\gamma \in \Pi_{g, n}$, defines a homomorphism $\widetilde{J}_{n}(f): \Pi_{g, n} \rightarrow W^{2} \Pi / W^{3} \Pi$, which factors through $H$. Let us denote by $\operatorname{Aut}_{H}\left(\Pi_{g, n}\right)$ the group of automorphisms of $\Pi_{g, n}$ inducing the identity on $H$. It is easy to check that the assignment $f \mapsto j_{n}(f)$ is a homomorphism as well. It then defines an abelian representation:

$$
\tilde{J}_{n}: \operatorname{Aut}_{H}\left(\Pi_{g, n}\right) \rightarrow \operatorname{Hom}\left(H, W^{2} \Pi / W^{3} \Pi\right) .
$$

Let us observe that $\operatorname{inn}\left(\Pi_{g, n}\right)$ is contained in $\operatorname{Aut}_{H}\left(\Pi_{g, n}\right)$. For $\alpha \in \Pi_{g, n}$, the homomorphism $\widetilde{J}_{n}($ inn $\alpha$ ) is defined by the assignment $\gamma \mapsto[\alpha, \gamma]$.

The composition $\widetilde{J}_{n} \circ$ inn: $\Pi_{g, n} \rightarrow \operatorname{Hom}\left(H, W^{2} \Pi / W^{3} \Pi\right)$ then factors through $H$ and its image is primitive in the codomain. Therefore, the quotient

$$
\operatorname{Hom}_{\mathrm{ext}}\left(H, W^{2} \Pi / W^{3} \Pi\right):=\operatorname{Hom}\left(H, W^{2} \Pi / W^{3} \Pi\right) / \widetilde{J}_{n}\left(\operatorname{inn}\left(\Pi_{g, n}\right)\right) .
$$

is torsion free.

Definition 3.17 The homomorphism $\widetilde{J}_{n}$ induces the abelian representation of the Torelli group:

$$
J_{n}: \mathcal{T}_{g, n} \rightarrow \operatorname{Hom}_{\text {ext }}\left(H, W^{2} \Pi / W^{3} \Pi\right),
$$

which we call Johnson's homomorphism and whose kernel coincides with that of the natural representation $\rho_{W^{3}}: \Gamma_{g, n} \rightarrow \operatorname{Out}\left(\Pi_{g, n} / W^{3} \Pi\right)$. 
From Theorem 3.15, it follows that the kernel of $J_{n}$ is precisely the Johnson's subgroup $\mathcal{K}_{g, n}$. In particular, ker $j_{i}=\operatorname{ker} J_{i}$ holds, for $i=0,1$. More importantly, the quotient $\mathcal{T}_{g, n} / \mathcal{K}_{g, n}:=I_{n}$ is a torsion free abelian group which we are going to determine explicitly. By Theorem 3.12, there is an exact commutative diagram:

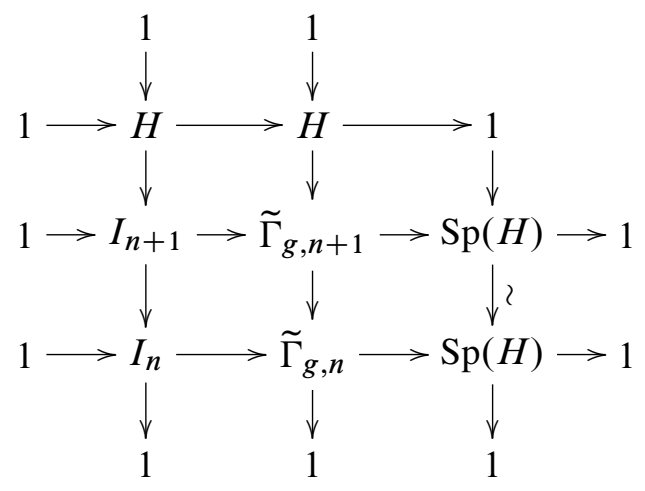

Let us remark that the middle and then the left column admit $n$ natural $\operatorname{Sp}(H)-$ equivariant splittings. A simple induction on $n$ then yields:

Theorem 3.18 The quotient $\mathcal{T}_{g, n} / \mathcal{K}_{g, n}$ is a free abelian group.

(i) For $g=1$ and $n \geq 1$, it has rank $2(n-1)$. So there is a split short exact sequence:

$$
1 \rightarrow \mathbb{Z}^{2(n-1)} \rightarrow \widetilde{\Gamma}_{1, n} \rightarrow \mathrm{SL}_{2}(\mathbb{Z}) \rightarrow 1
$$

(ii) For $g \geq 2$, it has rank $\left(\begin{array}{c}2 g \\ 3\end{array}\right)+2 g(n-1)$. So there is a short exact sequence:

$$
1 \rightarrow \mathbb{Z}^{\left(\begin{array}{c}
2 g \\
3
\end{array}\right)+2 g(n-1)} \rightarrow \widetilde{\Gamma}_{g, n} \rightarrow \operatorname{Sp}_{2 g}(\mathbb{Z}) \rightarrow 1
$$

Similar results hold for the profinite groups $\widetilde{\Gamma}_{g, n}^{\wedge}$ and $\widetilde{\Gamma}_{g, n}^{(\ell)}$.

Remark 3.19 Let $H^{\oplus n}$ and $\hat{H}^{\oplus n}$ denote the direct sum of $n$ copies of the first integral homology group of the compact Riemann surface $S_{g}$ and of its profinite completion. Let then the groups $\widetilde{\Gamma}_{g, 1}$ and $\widetilde{\Gamma}_{g, 1}^{\wedge}$, for $g \geq 1$, act on them via their natural representations in the symplectic groups $\operatorname{Sp}(H)$ and $\operatorname{Sp}(\hat{H})$. Theorem 3.18 and Theorem 3.12 yield, for $g \geq 1$, natural isomorphisms:

$$
\tilde{\Gamma}_{g, n+1} \cong H^{\oplus n} \rtimes \widetilde{\Gamma}_{g, 1} \quad \text { and } \quad \tilde{\Gamma}_{g, n+1}^{\wedge} \cong \hat{H}^{\oplus n} \rtimes \widetilde{\Gamma}_{g, 1}^{\wedge} .
$$

In particular, it follows that the groups $\widetilde{\Gamma}_{g, n}$ are linear for $g=1$ and $n \geq 1$. Below, we will see that this is the case for all $2 g-2+n>0$. 
In [21] (see also Section 2 of [22]), Morita extended Johnson's homomorphisms $j_{0}$ and $j_{1}$ to the whole Teichmüller group. More precisely, let $\frac{1}{2} \wedge^{3} H:=\wedge^{3} H \otimes \mathbb{Z}[1 / 2]$. There is a natural action of the symplectic group $\operatorname{Sp}(H)$ on $\frac{1}{2} \wedge^{3} H$. By means of this action, let us define semidirect products:

$$
\frac{1}{2} \wedge^{3} H \rtimes \operatorname{Sp}(H) \text { and } \frac{1}{2} \wedge^{3} H /(\omega \wedge H) \rtimes \operatorname{Sp}(H),
$$

where $\omega$ denotes the orientation class in $H_{2}\left(S_{g}\right)$. For $g \geq 2$, Morita extended the Johnson's homomorphisms to natural linear representations:

$$
m_{0}: \Gamma_{g} \rightarrow \frac{1}{2} \wedge^{3} H /(\omega \wedge H) \rtimes \operatorname{Sp}(H) \quad \text { and } \quad m_{1}: \Gamma_{g, 1} \rightarrow \frac{1}{2} \wedge^{3} H \rtimes \operatorname{Sp}(H),
$$

whose images are respectively $\widetilde{\Gamma}_{g}$ and $\widetilde{\Gamma}_{g, 1}$ and have finite index. In particular, both $\widetilde{\Gamma}_{g}$ and $\widetilde{\Gamma}_{g, 1}$ are linear groups. Combining Morita's results with Remark 3.19, we conclude that this holds true, for $2 g-2+n>0$, for all the groups $\widetilde{\Gamma}_{g, n}$. More precisely, we obtain the following generalization of Theorem 4.8 in [21]:

Theorem 3.20 For $g \geq 2$, the topological fundamental group of $\widetilde{\mathcal{M}}_{g, n+1}$ admits a natural embedding in the linear group $\left(H^{\oplus n} \oplus \frac{1}{2} \wedge^{3} H\right) \rtimes \operatorname{Sp}(H)$ as a subgroup of finite index.

Let $2 g-2+n>0$ and $g \geq 1$. Consider the following exact commutative diagram:

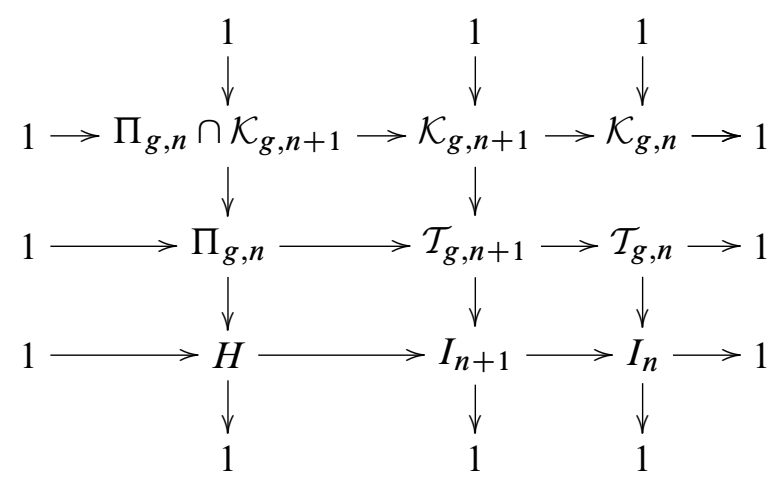

In the canonical morphism $\Pi_{g, n} \hookrightarrow \Gamma_{g, n+1}$, obtained, with the notation of Section 2, identifying $\Pi_{g, n}$ with the fundamental group of $S_{g} \backslash\left\{P_{1}, \ldots, P_{n}\right\}$ based at $P_{n+1}$, a small loop around the puncture $P_{i}$, for $i=1, \ldots, n$, is sent to the Dehn twist along a simple s.c.c. bounding the 2 -punctured disc containing $P_{i}$ and $P_{n+1}$ in $S_{g, n}$. Therefore, all such elements are contained in $\Pi_{g, n} \cap \mathcal{K}_{g, n+1}$. Thus, passing to 
abelianizations, we obtain (here, for a given group $G$, we denote its abelianization by $\left.H_{1}(G)\right)$ :

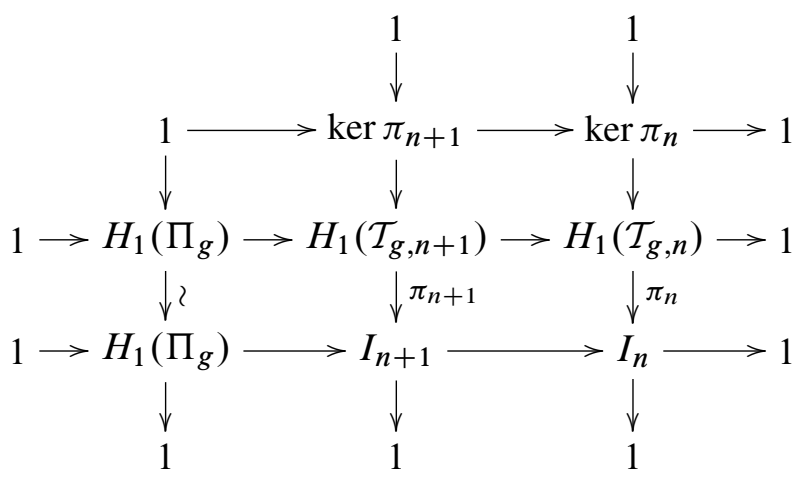

As we already remarked, the short exact sequence $1 \rightarrow H_{1}\left(\Pi_{g}\right) \rightarrow I_{n+1} \rightarrow I_{n} \rightarrow 1$ admits $n$ natural splittings in the category of $\operatorname{Sp}_{2 g}(\mathbb{Z})$-modules. Therefore, the same holds for all the short exact sequences in the above diagram. Since $I_{1}$ and $I_{0}$ are, in any case, free abelian groups, all the short exact sequences in the above commutative diagram split in the category of abelian groups for $n=0$ as well. Therefore, we have:

Theorem 3.21 Let $2 g-2+n>0$ and $g \geq 1$. For all $n \geq 0$, there is a natural short exact sequence of $\mathrm{Sp}_{2 g}(\mathbb{Z})$-modules which, if $n>0$, admits $n$ natural splittings:

$$
1 \rightarrow H_{1}\left(\Pi_{g}, \mathbb{Z}\right) \rightarrow H_{1}\left(\mathcal{T}_{g, n+1}, \mathbb{Z}\right) \rightarrow H_{1}\left(\mathcal{T}_{g, n}, \mathbb{Z}\right) \rightarrow 1 .
$$

In particular, there is a natural isomorphism of $\operatorname{Sp}_{2 g}(\mathbb{Z})$-modules:

$$
H_{1}\left(\mathcal{T}_{g, n+1}, \mathbb{Z}\right) \cong H_{1}\left(\mathcal{T}_{g, 1}, \mathbb{Z}\right) \oplus H_{1}\left(\Pi_{g}, \mathbb{Z}\right)^{\oplus n} .
$$

In the category of abelian groups, the short exact sequence above splits also for $n=0$.

Remark 3.22 Theorem 3.21, together with Johnson's computation of $H_{1}\left(\mathcal{T}_{g, n}, \mathbb{Z}\right)$, for $g \geq 3$ and $n=0,1$, in [14] and Mess' computation of $H_{1}\left(\mathcal{T}_{2}, \mathbb{Z}\right)$ in [18] provides a complete description of the abelianization of $\mathcal{T}_{g, n}$ for all $2 g-2+n>0$.

\section{The effect of clutching morphisms on fundamental groups}

Let $\sigma:=\left\{\gamma_{1}, \ldots, \gamma_{k}\right\}$ be a set of distinct, nontrivial, isotopy classes of separating s.c.c. on $S_{g, n}$, such that they admit a set of disjoint representatives none of them bounding a disc with a single puncture. There is then a homeomorphism $S_{g, n} \backslash \sigma \cong \coprod_{i=0}^{k} S_{g_{i}, n_{i}+1}$, where $\sum g_{i}=g, \sum n_{i}=n$ and $2 g_{i}-1+n_{i}>0$ for $i=0, \ldots, k$. Every such set $\sigma$ determines a clutching morphism, which factors through an étale cover of its image:

$$
\partial_{\sigma}: \widetilde{\mathcal{M}}_{g_{0}, n_{0}+1} \times \ldots \times \widetilde{\mathcal{M}}_{g_{k}, n_{k}+1} \rightarrow \widetilde{\mathcal{M}}_{g, n} .
$$


This map is an embedding, except for $n=0, k=1$ and $g_{0}=g_{1}$, when it factors through a $\mathbb{Z} / 2$-cover of its image. The image of $\partial_{\sigma}$ is the closed irreducible substack of $\widetilde{\mathcal{M}}_{g, n}$ who generically parametrizes singular curves homeomorphic to the surface obtained collapsing on $S_{g, n}$ the s.c.c. in the set $\sigma$.

The aim of this section is to describe the effect of the above natural morphisms on fundamental groups.

Theorem 4.1 Let $2 g-2+n>0$ and let $\sigma$ be a set of s.c.c. on $S_{g, n}$ as above. The clutching morphism $\partial_{\sigma}$ induces on topological fundamental groups a monomorphism:

$$
\partial_{\sigma *}: \pi_{1}\left(\widetilde{\mathcal{M}}_{g_{0}, n_{0}+1}\right) \times \ldots \times \pi_{1}\left(\widetilde{\mathcal{M}}_{g_{k}, n_{k}+1}\right) \hookrightarrow \pi_{1}\left(\widetilde{\mathcal{M}}_{g, n}\right) .
$$

Proof It is clearly enough to prove the theorem for $k=1$. Let then $\gamma$ be a separating s.c.c. on $S_{g, n}$ such that there is a homeomorphism $S_{g, n} \backslash \gamma \cong S_{g_{0}, n_{0}+1} \amalg S_{g_{1}, n_{1}+1}$. In order to prove that the induced homomorphism $\partial_{\gamma *}$ is injective, we need to introduce some notation.

Let $\widehat{\mathcal{M}}_{g, n}$ be the real oriented blow-up of $\widetilde{\mathcal{M}}_{g, n}$ along the divisor $\widetilde{\mathcal{M}}_{g, n} \backslash \mathcal{M}_{g, n}$ (more details on this construction can be found in Section 3 of Boggi [4]). There is a natural embedding $\mathcal{M}_{g, n} \hookrightarrow \widehat{\mathcal{M}}_{g, n}$ which is a homotopy equivalence. So the natural projection $\widehat{\mathcal{M}}_{g, n} \rightarrow \widehat{\mathcal{M}}_{g, n}$ induces a short exact sequence:

$$
1 \rightarrow \mathcal{K}_{g, n} \rightarrow \pi_{1}\left(\widehat{\mathcal{M}}_{g, n}\right) \rightarrow \pi_{1}\left(\widetilde{\mathcal{M}}_{g, n}\right) \rightarrow 1 .
$$

Let $\widehat{\Delta}_{\gamma} \rightarrow \widehat{\mathcal{M}}_{g, n}$ be the pullback of the clutching morphism

$$
\mathcal{M}_{g_{0}, n_{0}+1} \times \mathcal{M}_{g_{1}, n_{1}+1} \rightarrow \widetilde{\mathcal{M}}_{g, n}
$$

along the projection $\widehat{\mathcal{M}}_{g, n} \rightarrow \widetilde{\mathcal{M}}_{g, n}$. The morphism $\widehat{\Delta}_{\gamma} \rightarrow \widehat{\mathcal{M}}_{g, n}$ induces a monomorphism $\pi_{1}\left(\widehat{\Delta}_{\gamma}\right) \hookrightarrow \Gamma_{g, n}$ on fundamental groups, which identifies $\pi_{1}\left(\widehat{\Delta}_{\gamma}\right)$ with the stabilizer $\Gamma_{\vec{\gamma}}$ of the oriented isotopy class of the s.c.c. $\gamma$ on $S_{g, n}$, for the natural action of the mapping class group $\Gamma_{g, n}$. The natural projection $\widehat{\Delta}_{\gamma} \rightarrow \mathcal{M}_{g_{0}, n_{0}+1} \times \mathcal{M}_{g_{1}, n_{1}+1}$ is the $S^{1}$-bundle inducing the classical central extension which describes the stabilizer $\Gamma_{\vec{\gamma}} \cong \pi_{1}\left(\widehat{\Delta}_{\gamma}\right)$ for the action of $\Gamma_{g, n}$ on oriented s.c.c.:

$$
1 \rightarrow \mathbb{Z} \cdot \tau_{\gamma} \rightarrow \pi_{1}\left(\widehat{\Delta}_{\gamma}\right) \stackrel{p}{\rightarrow} \Gamma_{g_{0}, n_{0}+1} \times \Gamma_{g_{1}, n_{1}+1} \rightarrow 1 .
$$

There is also a short exact sequence:

$$
1 \rightarrow \mathcal{K}_{g_{0}, n_{0}+1} \times \mathcal{K}_{g_{1}, n_{1}+1} \rightarrow \Gamma_{g_{0}, n_{0}+1} \times \Gamma_{g_{1}, n_{1}+1} \rightarrow \widetilde{\Gamma}_{g_{0}, n_{0}+1} \times \widetilde{\Gamma}_{g_{1}, n_{1}+1} \rightarrow 1
$$


From the commutative diagram

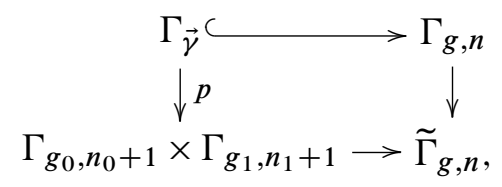

it then follows that, in order to prove the theorem, all we need to show is that

$$
p\left(\Gamma_{\vec{\gamma}} \cap \mathcal{K}_{g, n}\right)=\mathcal{K}_{g_{0}, n_{0}+1} \times \mathcal{K}_{g_{1}, n_{1}+1} .
$$

The inclusion $\mathcal{K}_{g_{0}, n_{0}+1} \times \mathcal{K}_{g_{1}, n_{1}+1} \leq p\left(\Gamma_{\vec{\gamma}} \cap \mathcal{K}_{g, n}\right)$ being trivial, it is enough to prove that:

$$
p\left(\Gamma_{\vec{\gamma}} \cap \mathcal{K}_{g, n}\right) \leq \mathcal{K}_{g_{0}, n_{0}+1} \times \mathcal{K}_{g_{1}, n_{1}+1} .
$$

Let $f \in \Gamma_{\vec{\gamma}}$, it can be represented by the product of two commuting homeomorphisms $f_{0}$ and $f_{1}$, supported respectively on $S_{g_{0}, n_{0}+1}$ and $S_{g_{1}, n_{1}+1}$. It is easily checked that $f \in \mathcal{T}_{g, n} \geq \mathcal{K}_{g, n}$ if and only if $p\left(f_{i}\right) \in \mathcal{T}_{g_{i}, n_{i}+1}$, for $i=0,1$. Therefore, if $f \in \Gamma_{\vec{\gamma}} \cap \mathcal{K}_{g, n}$, we already know that $p\left(f_{i}\right) \in \mathcal{T}_{g_{i}, n_{i}+1}$, for $i=0$, 1 . Let, as usual, $H:=H_{1}\left(S_{g}\right)$ and let then $H_{i}:=H_{1}\left(S_{g_{i}, n_{i}+1}\right)$, for $i=0,1$. Thus, in order to prove that actually $p\left(f_{i}\right) \in \mathcal{K}_{g_{i}, n_{i}+1}$, for $i=0$, 1 , by Theorem 3.15 , it is enough to show that, since $f$ is in the kernel of the Johnson's homomorphism (see Definition 3.17):

$$
J_{n}: \mathcal{T}_{g, n} \rightarrow \operatorname{Hom}_{\mathrm{ext}}\left(H, W^{2} \Pi / W^{3} \Pi\right),
$$

then the homomorphisms $p\left(f_{i}\right)$ are in the kernel of the Johnson's homomorphisms:

$$
J_{n_{i}+1}: \mathcal{T}_{g_{i}, n_{i}+1} \rightarrow \operatorname{Hom}_{\mathrm{ext}}\left(H_{i}, W^{2} \Pi / W^{3} \Pi\right), \quad \text { for } i=0,1 .
$$

The embeddings $S_{g_{i}, n_{i}+1} \hookrightarrow S_{g, n}$ induce monomorphisms $\Pi_{g_{i}, n_{i}+1} \hookrightarrow \Pi_{g, n}$, for $i=0,1$. From Lemma 2.5 in [6], more directly, from its proof, it follows that

$$
\Pi_{g_{i}, n_{i}+1} \cap W^{3} \Pi_{g, n}=W^{3} \Pi_{g_{i}, n_{i}+1}, \quad \text { for } i=0,1 .
$$

Therefore, for $i=0,1$, there are monomorphisms $\Pi_{g_{i}, n_{i}+1} / W^{3} \Pi \hookrightarrow \Pi_{g, n} / W^{3} \Pi$ which fit in the commutative diagrams with exact rows:

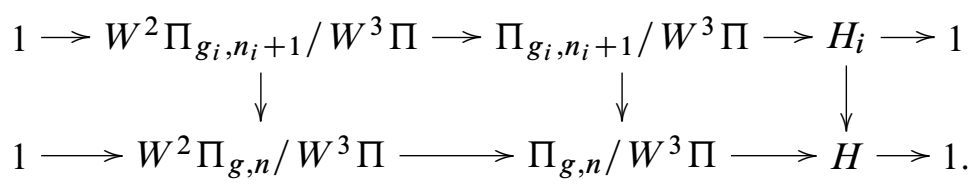

So the homomorphism $\widetilde{J}_{n}(f): H \rightarrow W^{2} \Pi / W^{3} \Pi$ induces by restriction the two homomorphism $\widetilde{J}_{n_{i}+1}\left(p\left(f_{i}\right)\right)$, for $i=0,1$. Now, $f \in \operatorname{ker} J_{n}$ if and only if $\widetilde{J}_{n}(f)=$ $\left[\alpha,{ }_{-}\right]$for some $\alpha \in \Pi_{g, n}$. On the other hand, as we remarked in Section 3, the composition $\widetilde{J}_{n}\left(\right.$ inn $\alpha$ ) only depends on the image $\bar{\alpha} \in H=H_{0} \oplus H_{1}$. Since $f \in \Gamma_{\vec{\gamma}}$, 
for $i=0,1$, it follows that $[\alpha, \beta] \in W^{2} \Pi_{g_{i}, n_{i}+1} / W^{3} \Pi$ for all $\beta \in H_{i}$. It is then easy to see that $\bar{\alpha} \in H_{0} \cap H_{1}=\{0\}$ and so that $f_{i} \in \operatorname{ker} J_{n_{i}+1}$, for $i=0,1$, as well.

The construction of the Johnson's homomorphism has a profinite and a pro- $\ell$ analogue. Let us sketch the profinite case. The profinite group $\widehat{\Pi}_{g, n} / W^{3} \widehat{\Pi}$ is an extension of torsion free abelian groups:

$$
1 \rightarrow W^{2} \widehat{\Pi} / W^{3} \widehat{\Pi} \rightarrow \widehat{\Pi}_{g, n} / W^{3} \widehat{\Pi} \rightarrow \widehat{H} \rightarrow 1 .
$$

Proceeding like in Section 3 (Definition 3.17), we define the abelian representations:

$$
\begin{aligned}
& \tilde{J}_{n}^{\wedge}: \operatorname{Aut}_{\widehat{H}}\left(\widehat{\Pi}_{g, n}\right) \rightarrow \operatorname{Hom}\left(\widehat{H}, W^{2} \widehat{\Pi} / W^{3} \widehat{\Pi}\right), \\
& \widehat{J}_{n}: \widehat{\mathcal{T}}_{g, n} \rightarrow \operatorname{Hom}_{\text {ext }}\left(H, W^{2} \Pi / W^{3} \Pi\right) .
\end{aligned}
$$

From the isomorphisms $W^{2} \widehat{\Pi} / W^{3} \widehat{\Pi} \cong\left(W^{2} \Pi / W^{3} \Pi\right) \otimes \widehat{\mathbb{Z}}$ and $\hat{H} \cong H \otimes \widehat{\mathbb{Z}}$, it follows that the embeddings $S_{g_{i}, n_{i}+1} \hookrightarrow S_{g, n}$ induce monomorphisms

$$
\widehat{\Pi}_{g_{i}, n_{i}+1} / W^{3} \widehat{\Pi} \hookrightarrow \widehat{\Pi}_{g, n} / W^{3} \widehat{\Pi},
$$

for $i=0,1$, and that, moreover, there are natural isomorphisms:

$$
\begin{aligned}
& \operatorname{Hom}\left(\widehat{H}, W^{2} \widehat{\Pi} / W^{3} \widehat{\Pi}\right) \cong \operatorname{Hom}\left(H, W^{2} \Pi / W^{3} \Pi\right) \otimes \widehat{\mathbb{Z}} \\
& \widetilde{J}_{n}^{\wedge}\left(\operatorname{inn}\left(\widehat{\Pi}_{g, n}\right)\right) \cong \widetilde{J}_{n}\left(\operatorname{inn}\left(\Pi_{g, n}\right)\right) \otimes \widehat{\mathbb{Z}} .
\end{aligned}
$$

The same argument of the proof of Theorem 4.1 then yields the following algebraic version:

Theorem 4.2 Let $2 g-2+n>0$ and let $\sigma$ be a set of s.c.c. on $S_{g, n}$ as above. The clutching morphism $\partial_{\sigma}$ induces on algebraic fundamental groups a homomorphism:

$$
\widehat{\partial}_{\sigma *}: \hat{\pi}_{1}\left(\widetilde{\mathcal{M}}_{g_{0}, n_{0}+1}\right) \times \ldots \times \hat{\pi}_{1}\left(\widetilde{\mathcal{M}}_{g_{k}, n_{k}+1}\right) \hookrightarrow \hat{\pi}_{1}\left(\widetilde{\mathcal{M}}_{g, n}\right),
$$

which is injective for $g \leq 1$ and for $g_{i} \neq 1$, for all $i=0, \ldots, k$. If $g_{i_{j}}=1$ for $0 \leq i_{j} \leq k$ and $j=1, \ldots, h$, then the kernel of $\widehat{\partial}_{\sigma *}$ is the product of the subgroups $\widehat{F}_{\infty}$ contained in the factors $\widehat{\pi}_{1}\left(\widetilde{\mathcal{M}}_{1, n_{i j}+1}\right)$, for $j=1, \ldots k$. A similar result holds for the profinite groups $\widetilde{\Gamma}_{g, n}^{(\ell)}$, for all primes $\ell>0$.

Let us remark the analogy with the properties of the natural stratification of the moduli stack $\mathcal{A}_{g}$ of principally polarized complex abelian varieties of dimension $g$, given by the loci of decomposable abelian varieties. For every couple of positive integers $g_{1}$ and $g_{2}$ such that $g_{1}+g_{2}=g$, there is indeed a natural morphisms of D-M stacks:

$$
\mathcal{A}_{g_{1}} \times \mathcal{A}_{g_{2}} \rightarrow \mathcal{A}_{g}
$$


which is étale on the image and an embedding if and only if $g_{1} \neq g_{2}$. The homomorphism induced on topological fundamental groups is always a monomorphism, corresponding to the natural monomorphism $\operatorname{Sp}_{2 g_{1}}(\mathbb{Z}) \times \operatorname{Sp}_{2 g_{2}}(\mathbb{Z}) \hookrightarrow \operatorname{Sp}_{2 g}(\mathbb{Z})$. The homomorphism induced on algebraic fundamental groups is a monomorphism if $g_{i} \neq 1$, for both $i=1,2$. Otherwise, its kernel is the product of the kernels of the natural epimorphisms $\widehat{\mathrm{Sp}}_{2 g_{i}}(\mathbb{Z}) \rightarrow \mathrm{Sp}_{2 g_{i}}(\widehat{\mathbb{Z}})$, for $i=1,2$, which is nontrivial if and only if $g_{i}=1$.

A significant difference between the moduli stacks $\widetilde{\mathcal{M}}_{g, n}$ and the moduli stacks $\mathcal{A}_{g}$ is the fact that $\pi_{1}\left(\mathcal{A}_{g}\right)$ is nontrivial whenever $\operatorname{dim} \mathcal{A}_{g}>0$ while $\pi_{1}\left(\widetilde{\mathcal{M}}_{0, n}\right)=\{1\}$ for all $n \geq 3$. In particular, the moduli space $\widetilde{\mathcal{M}}_{0,4}=\overline{\mathcal{M}}_{0,4}$ is a sphere, from which, by standard arguments (see Proposition A.4 of Mondello [20]), it follows that the moduli stack of curves of compact type $\widetilde{\mathcal{M}}_{g, n}$ is not a classifying space for the group $\widetilde{\Gamma}_{g, n}$, for $g=2$ and $n \geq 2, g=3$ and $n \geq 1$, and for all $g>3$.

More precisely, in [20], it is proved that the stack $\widetilde{\mathcal{M}}_{g, n}$ is a classifying space if and only if $(g, n)=(0,3),(1,1),(2,0)$. This is in sharp contrast with the case of the stack $\mathcal{A}_{g}$, which is a classifying space for the group $\operatorname{Sp}_{2 g}(\mathbb{Z})$ for all $g \geq 1$.

\section{On the cohomology of $\widetilde{\Gamma}_{g, n}$}

The Teichmüller space $T_{g, n}$ is contractible, hence the cohomology of the group $\Gamma_{g, n}$ is naturally isomorphic to the cohomology of the space $\mathcal{M}_{g, n}$.

Let us define some natural cohomology classes on the Deligne-Mumford compactification $\overline{\mathcal{M}}_{g, n}$. By restriction, we will then get cohomology classes for $\mathcal{M}_{g, n}$ and so for the Teichmüller group $\Gamma_{g, n}$.

The morphism $p: \overline{\mathcal{M}}_{g, n+1} \rightarrow \overline{\mathcal{M}}_{g, n}$ (forgetting the last labeled point) is naturally identified with the universal curve. Let $\omega_{p}$ be its relative dualizing line bundle, let the $s_{i}$, for $i=1, \ldots, n$ be the tautological sections of $p$ and let $L_{i}:=s_{i}^{*}\left(\omega_{p}\right)$.

The first Chern class of the line bundle $L_{i}$, which is denoted by $\psi_{i}$, is a cohomology class in $H^{2}\left(\overline{\mathcal{M}}_{g, n}, \mathbb{Q}\right)$, for $i=1, \ldots, n$. Then, for $r \geq 0$, let

$$
\kappa_{r}:=\pi_{*}\left(\psi_{n+1}^{r+1}\right) \in H^{2 r}\left(\overline{\mathcal{M}}_{g, n}, \mathbb{Q}\right) .
$$

The $\psi_{i}$, for $i=1, \ldots, n$, together with the $\kappa_{r}$, for $r \geq 0$, are called tautological classes. Let us denote in the same way their restrictions to $\mathcal{M}_{g, n}$. They span a subalgebra of the cohomology ring $H^{*}\left(\mathcal{M}_{g, n}, \mathbb{Q}\right)$, called the tautological algebra. All geometrically relevant cohomology classes are contained there. 
In fact, much more is true. Harer [10] proved that the cohomology of $\Gamma_{g, n}$ stabilizes in degrees less or equal than $(g-1) / 2$. Recently, in a spectacular breakthrough, following a conjecture of Mumford, Madsen and Weiss [17] proved that, in the stable range, all the rational cohomology of $\mathcal{M}_{g, n}$ coincides with the tautological algebra.

The natural epimorphism $\Gamma_{g, n} \rightarrow \widetilde{\Gamma}_{g, n}$ induces a homomorphism of cohomology rings $H^{*}\left(\widetilde{\Gamma}_{g, n}\right) \rightarrow H^{*}\left(\Gamma_{g, n}\right)$. It is then natural to ask whether the tautological algebra is in the image of this homomorphism. As we are going to show, from some results of Morita, it follows that this is the case for $g \geq 2$ and all $n \geq 0$. This is in contrast with the case of the natural epimorphism $\Gamma_{g, n} \rightarrow \mathrm{Sp}_{2 g}(\mathbb{Z})$. In fact, the image of the induced homomorphism $H^{*}\left(\operatorname{Sp}_{2 g}(\mathbb{Z})\right) \rightarrow H^{*}\left(\Gamma_{g, n}\right)$ contains only the classes $\kappa_{2 i}$, for $i \geq 0$.

Let us review Morita's results. Denote respectively by $U_{g}$ and $U_{g, 1}$ the abelian groups $\frac{1}{2} \wedge^{3} H /(\omega \wedge H)$ and $\frac{1}{2} \wedge^{3} H$ defined in Section 3. In [22], Morita showed that, for $g \geq 2$ and $n=0,1$, there is a natural homomorphism of cohomology rings

$$
H^{*}\left(U_{g, n}, \mathbb{Q}\right)^{\mathrm{Sp}} \rightarrow H^{*}\left(U_{g, n} \rtimes \mathrm{Sp}_{2 g}(\mathbb{Z}), \mathbb{Q}\right),
$$

which, combined with the homomorphism

$$
H^{*}\left(U_{g, n} \rtimes \mathrm{Sp}_{2 g}(\mathbb{Z}), \mathbb{Q}\right) \rightarrow H^{*}\left(\Gamma_{g, n}, \mathbb{Q}\right),
$$

induced by Morita's extension of Johnson's homomorphism, provides a homomorphism of cohomology rings

$$
H^{*}\left(U_{g, n}, \mathbb{Q}\right)^{\mathrm{Sp}} \rightarrow H^{*}\left(\Gamma_{g, n}, \mathbb{Q}\right),
$$

whose image contains the tautological algebra. In particular, since the above homomorphism factors through the natural homomorphism $H^{*}\left(\widetilde{\Gamma}_{g, n}, \mathbb{Q}\right) \rightarrow H^{*}\left(\Gamma_{g, n}, \mathbb{Q}\right)$, it follows that the image of the latter contains as well the tautological algebra, for $n=0,1$. Morita's result easily extends to the $n$-pointed case, for all $n \geq 0$ :

\section{Proposition 5.1 Let $g \geq 2$.}

(i) The natural homomorphism $\Gamma_{g, n} \rightarrow \widetilde{\Gamma}_{g, n}$ induces a homomorphism on cohomology rings

$$
H^{*}\left(\widetilde{\Gamma}_{g, n}, \mathbb{Q}\right) \rightarrow H^{*}\left(\Gamma_{g, n}, \mathbb{Q}\right),
$$

whose image contains the tautological algebra.

(ii) The natural homomorphism $\Gamma_{g, n} \rightarrow \widetilde{\Gamma}_{g, n}^{(\ell)}$ induces a homomorphism of the $\ell$-adic cohomology rings

$$
H^{*}\left(\tilde{\Gamma}_{g, n}^{(\ell)}, \mathbb{Q}_{\ell}\right) \rightarrow H^{*}\left(\Gamma_{g, n}, \mathbb{Q}_{\ell}\right),
$$

whose image contains the tautological algebra. 
Proof The first item of the proposition has been proved for $n=0,1$. For $n>1$, let us consider the natural epimorphisms $p_{i}: \Gamma_{g, n} \rightarrow \Gamma_{g, 1}$, corresponding to filling in the set of punctures $\left\{P_{1}, \ldots, P_{n}\right\} \backslash\left\{P_{i}\right\}$ on $S_{g, n}$, for $i=1, \ldots, n$. Let then $S_{g, n}^{i}$ be the Riemann surface with boundary obtained from $S_{g, n}$ removing a small open disc $D_{i}$ containing $P_{i}$ and let us denote by $\Gamma\left(S_{g, n}^{i}\right)$ the mapping class group of $S_{g, n}^{i}$, for $i=1, \ldots, n$. There is a natural epimorphism $\Gamma\left(S_{g, n}^{i}\right) \rightarrow \Gamma_{g, n}$ with kernel generated by the Dehn twist along the s.c.c. $\partial D_{i}$. Then the class $\psi_{i} \in H^{2}\left(\Gamma_{g, n}, \mathbb{Q}\right)$, for $i=1, \ldots, n$, corresponds to the central extension:

$$
1 \rightarrow \mathbb{Z} \rightarrow \Gamma\left(S_{g, n}^{i}\right) \rightarrow \Gamma_{g, n} \rightarrow 1 .
$$

It is clear that such extension is just the pullback along $p_{i}$ of the extension:

$$
1 \rightarrow \mathbb{Z} \rightarrow \Gamma\left(S_{g, 1}^{1}\right) \rightarrow \Gamma_{g, 1} \rightarrow 1,
$$

ie that $\psi_{i}=p_{i}^{*}\left(\psi_{1}\right)$, for $i=1, \ldots, n$. For $i=1, \ldots, n$, there is a commutative diagram:

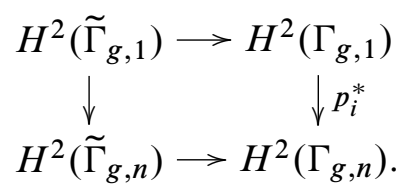

Since the image of $H^{2}\left(\widetilde{\Gamma}_{g, 1}\right)$ in $H^{2}\left(\Gamma_{g, 1}\right)$ contains $\psi_{1}$, it follows that $p_{i}^{*}\left(\psi_{1}\right)=\psi_{i}$ is in the image of $H^{2}\left(\widetilde{\Gamma}_{g, n}\right)$ in $H^{2}\left(\Gamma_{g, n}\right)$, for $i=1, \ldots, n$.

As to the classes $\kappa_{r}$, for $r \geq 1$, letting $p: \Gamma_{g, n+1} \rightarrow \Gamma_{g, n}$ be the epimorphism corresponding to filling in the $n+1$-th puncture on $S_{g, n+1}$, the identity $p^{*}\left(\kappa_{1}\right)=\kappa_{1}-\psi_{n+1}$ holds in $H^{2}\left(\Gamma_{g, n+1}\right)$ and, more in general, $p^{*}\left(\kappa_{r}\right)$ equals the sum of the class $\kappa_{r}$ in $H^{2 r}\left(\Gamma_{g, n+1}\right)$ plus a polynomial in lower degree tautological classes (this is proved in Arbarello and Cornalba [1]). Therefore, a simple induction and an argument similar to the above yields that $\kappa_{r} \in H^{*}\left(\Gamma_{g, n}\right)$ is in the image of $H^{*}\left(\widetilde{\Gamma}_{g, n}\right)$, for all $r \geq 1$, thus completing the proof of item (i) of the theorem.

In order to prove item (i), we just need to remark the following. Let $U_{g, n}^{(\ell)}$, for $n=0,1$, be the pro- $\ell$ completion of the finitely generated abelian group $U_{g, n}$. The natural homomorphism $U_{g, n} \rightarrow U_{g, n}^{(\ell)}$ then induces an isomorphism on $\ell$-adic cohomology $H^{*}\left(U_{g, n}^{(\ell)}, \mathbb{Q}_{\ell}\right) \cong H^{*}\left(U_{g, n}, \mathbb{Q}_{\ell}\right)$, for $n=0,1$. The argument produced to prove (i) then extends to the $\ell$-adic case.

Remark 5.2 In Kawazumi and Morita [15], it is actually proved that the image of the natural homomorphism $H^{*}\left(\widetilde{\Gamma}_{g, n}, \mathbb{Q}\right) \rightarrow H^{*}\left(\Gamma_{g, n}, \mathbb{Q}\right)$, for $n=0,1$, in the stable range, equals the tautological algebra (this result is antecedent to the proof of Mumford's conjecture). 
Acknowledgements Motivated originally by the applications given in [5], this paper is an ideal continuation of my joint work with M Pikaart [6]. So my first thanks go to him and to E Looijenga with whom I shared a lot of ideas at the time of my doctorate in Utrecht. I thank also the referee of Geometry and Topology, whose careful reading and valuable remarks on a previous version of this paper allowed me to enhance the exposition and results.

\section{References}

[1] E Arbarello, M Cornalba, Combinatorial and algebro-geometric cohomology classes on the moduli spaces of curves, J. Algebraic Geom. 5 (1996) 705-749 MR1486986

[2] H Bass, J Milnor, J-P Serre, Solution of the congruence subgroup problem for $\mathrm{SL}_{n}(n \geq 3)$ and $\mathrm{Sp}_{2 n}(n \geq 2)$, Inst. Hautes Études Sci. Publ. Math. (1967) 59-137 MR0244257

[3] J S Birman, On Siegel's modular group, Math. Ann. 191 (1971) 59-68 MR0280606

[4] M Boggi, Profinite Teichmüller theory, Math. Nachr. 279 (2006) 953-987 MR2242960

[5] M Boggi, Monodromy of stable curves of compact type: rigidity and extension, Int. Math. Res. Not. IMRN (2007) 16pp Art. ID rnm017 MR2348091

[6] M Boggi, M Pikaart, Galois covers of moduli of curves, Compositio Math. 120 (2000) 171-191 MR1739177

[7] P Deligne, Le lemme de Gabber, Astérisque (1985) 131-150 MR801921 Seminar on arithmetic bundles: the Mordell conjecture (Paris, 1983/84)

[8] R Hain, Torelli groups and geometry of moduli spaces of curves, from: "Current topics in complex algebraic geometry (Berkeley, CA, 1992/93)", Math. Sci. Res. Inst. Publ. 28, Cambridge Univ. Press (1995) 97-143 MR1397061

[9] R Hain, E Looijenga, Mapping class groups and moduli spaces of curves, from: “Algebraic geometry-Santa Cruz 1995", Proc. Sympos. Pure Math. 62, Amer. Math. Soc. (1997) 97-142 MR1492535

[10] J L Harer, Stability of the homology of the mapping class groups of orientable surfaces, Ann. of Math. (2) 121 (1985) 215-249 MR786348

[11] D Johnson, An abelian quotient of the mapping class group $\mathcal{I}_{g}$, Math. Ann. 249 (1980) 225-242 MR579103

[12] D Johnson, The structure of the Torelli group. I. A finite set of generators for $\mathcal{I}$, Ann. of Math. (2) 118 (1983) 423-442 MR727699

[13] D Johnson, The structure of the Torelli group. II. A characterization of the group generated by twists on bounding curves, Topology 24 (1985) 113-126 MR793178 
[14] D Johnson, The structure of the Torelli group. III. The abelianization of $\mathcal{T}$, Topology 24 (1985) 127-144 MR793179

[15] N Kawazumi, S Morita, The primary approximation to the cohomology of the moduli space of curves and cocycles for the Mumford-Morita-Miller classes, University of Tokio pre-print 2001-13 (2001)

[16] F F Knudsen, The projectivity of the moduli space of stable curves. II. The stacks $M_{g, n}$, Math. Scand. 52 (1983) 161-199 MR702953

[17] I Madsen, MS Weiss, The stable moduli space of Riemann surfaces: Mumford's conjecture arXiv:math.AT/0212321v2

[18] G Mess, The Torelli groups for genus 2 and 3 surfaces, Topology 31 (1992) 775-790 MR1191379

[19] S Mochizuki, Extending families of curves over log regular schemes, J. Reine Angew. Math. 511 (1999) 43-71 MR1695789

[20] G Mondello, A remark on the homotopical dimension of some moduli spaces of stable Riemann surfaces, J. Eur. Math. Soc. (JEMS) 10 (2008) 231-241 MR2349902

[21] S Morita, The extension of Johnson's homomorphism from the Torelli group to the mapping class group, Invent. Math. 111 (1993) 197-224 MR1193604

[22] S Morita, A linear representation of the mapping class group of orientable surfaces and characteristic classes of surface bundles, from: "Topology and Teichmüller spaces (Katinkulta, 1995)”, World Sci. Publ., River Edge, NJ (1996) 159-186 MR1659679

[23] B Noohi, Foundations of topological stacks I arXiv:math. AG/0503247

[24] B Noohi, Fundamental groups of algebraic stacks, J. Inst. Math. Jussieu 3 (2004) 69-103 MR2036598

[25] M Pikaart, Moduli spaces of curves: stable cohomology and Galois covers, $\mathrm{PhD}$ thesis, Utrecht University (1997)

[26] M Pikaart, A J de Jong, Moduli of curves with non-abelian level structure, from: "The moduli space of curves (Texel Island, 1994)", Progr. Math. 129, Birkhäuser, Boston (1995) 483-509 MR1363068

[27] L Ribes, P Zalesskii, Profinite groups, Ergebnisse der Math. und ihrer Grenzgebiete. 3. Folge. [Results in Math. and Related Areas. 3rd Series.] A Series of Modern Surveys in Math. 40, Springer, Berlin (2000) MR1775104

Escuela de Matemática, Universidad de Costa Rica, Ciudad Universitaria Rodrigo Facio San Pedro de Montes de Oca, Apartado 2060, San José, Costa Rica marco.boggi@gmail.com

Proposed: Shigeyuki Morita Seconded: Joan Birman, Walter Neumann
Received: 4 December 2007 Revised: 22 May 2008 